Supporting Information (27 pages)

\title{
High Methane Storage Working Capacity in Metal-Organic Frameworks with Acrylate Links
}

\author{
Juncong Jiang, ${ }^{\dagger, \downarrow}$ Hiroyasu Furukawa, ${ }^{\dagger, \ddagger}$ Yue-Biao Zhang, ${ }^{\dagger}$ and Omar M. Yaghi ${ }^{\dagger, \square, *}$ \\ ${ }^{\dagger}$ Department of Chemistry, University of California-Berkeley, Materials Sciences \\ Division, Lawrence Berkeley National Laboratory, and Kavli Energy NanoSciences \\ Institute at Berkeley, Berkeley, California 94720, United States \\ ${ }^{\square}$ King Abdulaziz City for Science and Technology, Riyadh 11442, Saudi Arabia. \\ \$These authors contribute equally \\ *To whom correspondence should be addressed: Email, yaghi@berkeley.edu
}

\section{Table of Contents}

$\begin{array}{lll}\text { Section S1 } & \text { Single Crystal X-ray Diffraction Analyses } & \text { S2-S4 } \\ \text { Section S2 } & \text { Structure Models } & \text { S5-S9 } \\ \text { Section S3 } & \text { Powder X-ray Diffraction Patterns } & \text { S10-S12 } \\ \text { Section S4 } & \text { Thermogravimetric Analyses } & \text { S13-S15 } \\ \text { Section S5 } & \mathrm{N}_{2} \text { Adsorption Measurements } & \text { S16-S18 } \\ \text { Section S6 } & \text { Low Pressure } \mathrm{CH}_{4} \text { Adsorption Measurements } & \text { S19-S21 } \\ \text { Section S7 } & \text { High Pressure } \mathrm{CH}_{4} \text { Adsorption Measurements } & \text { S22-S24 } \\ \text { Section S8 } & \text { Complete List of Metal-Organic Frameworks with ith-d Net } & \text { S25-S26 } \\ \text { Section S9 } & \text { References } & \text { S27 }\end{array}$




\section{Section S1 Single Crystal X-ray Diffraction Analyses}

MOF-950. An as-synthesized crystal of MOF-950 was picked up from Paratone oil and mounted using a Kapton loop, before being placed under a nitrogen cold stream at $150 \mathrm{~K}$. The diffraction data were collected on a Bruker D8-Venture diffractometer equipped with Cu-target $(\lambda=1.54184 \AA)$ micro-focus X-ray tubes and a PHOTON 100 CMOS detector, operating at $50 \mathrm{~kW}$ and $1 \mathrm{~mA}$. The data were processed with the Bruker APEX2 software package, integrated using SAINT and scaled using SADABS. The structures were solved by direct methods and refined by full-matrix least squares on $F^{2}$. All the non-hydrogen atoms were refined anisotropically, and the hydrogen atoms were geometrically calculated and refined as riding atoms. The highly disordered guest molecules could not be modeled, so their contribution to diffraction was accounted for using the SQUEEZE routine in PLATON's software package. 
Table S1. Crystal data and structure refinement for MOF-950.

\begin{tabular}{|c|c|}
\hline Identification code & MOF-950 \\
\hline Empirical formula & $\mathrm{C}_{30} \mathrm{H}_{18} \mathrm{O}_{13} \mathrm{Zn}_{4}$ \\
\hline Formula weight & 848.00 \\
\hline Temperature / K & 150 \\
\hline Crystal system & cubic \\
\hline Space group & $P a-3$ \\
\hline$a / \AA$ & $21.2832(4)$ \\
\hline$\alpha /{ }^{\circ}$ & 90 \\
\hline Volume / $\AA^{3}$ & $9640.8(5)$ \\
\hline$Z$ & 4 \\
\hline$\rho_{\text {calc }} / \mathrm{mg} \mathrm{mm}^{-3}$ & 0.584 \\
\hline $\mathrm{m} / \mathrm{mm}^{-1}$ & 1.320 \\
\hline $\mathrm{F}(000)$ & 1688 \\
\hline Crystal size $/ \mathrm{mm}^{3}$ & $0.07 \times 0.09 \times 0.10$ \\
\hline Radiation & $\mathrm{CuK} \alpha(\lambda=1.54178 \AA)$ \\
\hline$\theta$ range & 3.6 to $49.7^{\circ}$ \\
\hline Index ranges & $-18 \leq h \leq 16,-13 \leq k \leq 21,-20 \leq l \leq 21$ \\
\hline Reflections collected & 15494 \\
\hline Unique reflections & 1633 \\
\hline Restraints/parameters & $6 / 96$ \\
\hline$R_{\text {int }}$ & 0.045 \\
\hline$R_{1}^{a}, w R_{2}^{b}$ & $0.0824,0.2495$ \\
\hline$S(\mathrm{GOF})^{c}$ & 1.005 \\
\hline Largest diff. peak/hole / e $\AA^{-3}$ & $0.36 /-0.44$ \\
\hline $\begin{array}{l}{ }^{a} R_{1}=\Sigma|| F_{\mathrm{o}}|-| F_{\mathrm{c}}|/ \Sigma| F_{\mathrm{o}} \mid ; \\
{ }^{b} w R_{2}=\left[\Sigma w\left(F_{\mathrm{o}}^{2}-F_{\mathrm{c}}^{2}\right)^{2} / \Sigma w\left(F_{\mathrm{o}}{ }^{2}\right.\right. \\
{ }^{c} S=\left[\Sigma w\left(F_{\mathrm{o}}^{2}-F_{\mathrm{c}}^{2}\right)^{2} /\left(N_{\mathrm{ref}}-N_{\mathrm{par}}\right.\right.\end{array}$ & \\
\hline
\end{tabular}




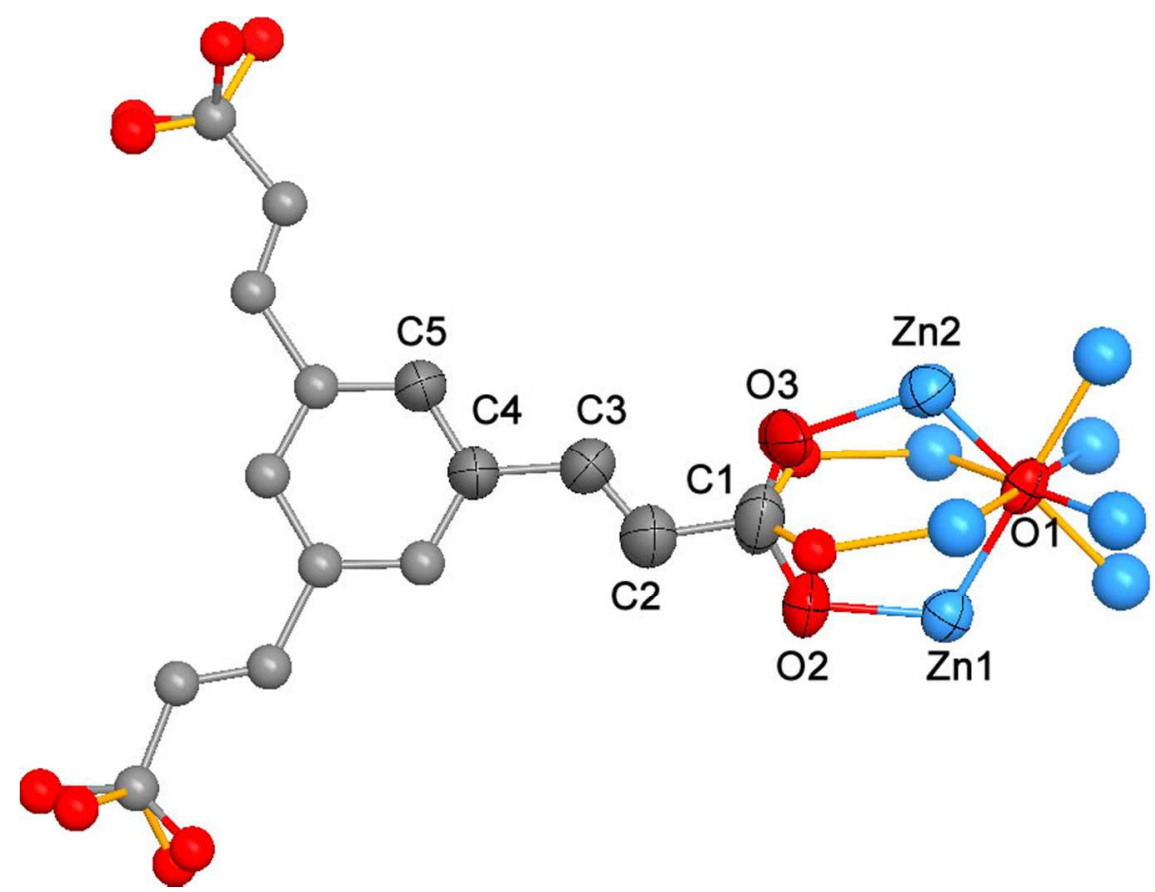

Figure S1. Asymmetric unit in the single crystal structure of MOF-950 (thermal ellipsoids with $30 \%$ probability). Hydrogen atoms are omitted for clarity; orange bonds represent the disordered set generated from symmetry. Symmetry-related atoms are not labeled and represented as spheres. 


\section{Section S2 Structure Models}

The models for MOF-905, MOF-905-Me 2 , MOF-905-Naph, and MOF-905-NO ${ }_{2}$, including cell parameters and atomic coordinates, were generated using Materials Studio 7.0 software employing the Crystal Building module. The crystal structures were generated based on that of MOF-205, but in the lower symmetry space group $P m \overline{3}$, considering the $C_{3}$-symmetry of the tritopic linker. The structures were energy minimized using the Forcite module in Materials Studio using the Geometry Optimization routine with a Universal force field (UFF). The unit cells were further optimized based on the Pawley fitting of experimental PXRD patterns (Section S4) with the Reflex module. Further geometry optimizations were performed for the tritopic linker to adapt the structure by modifying the twist-angle from the central benzene to the ethylene. The coordinates and the occupancy for the structures are listed in Tables S2-S5. 
Table S2. Crystallographic information and atomic fractional coordinates for the structure model of MOF-905.

\begin{tabular}{|c|c|c|c|c|}
\hline Identification Code & MOF-905 & & & \\
\hline Empirical Formula & $\mathrm{C}_{28} \mathrm{H}_{16} \mathrm{O}_{13} \mathrm{Zn}_{4}$ & & & \\
\hline Formula Weight & 821.97 & & & \\
\hline Crystal System & Cubic & & & \\
\hline Space Group & $P m \overline{3}$ & & & \\
\hline$a / \AA$ & 23.40810 & & & \\
\hline$V / \AA^{3}$ & 12826.2 & & & \\
\hline$Z$ & 6 & & & \\
\hline$d / \mathrm{g} \mathrm{cm}^{-3}$ & 0.638 & & & \\
\hline Atoms & $x$ & $y$ & $Z$ & Occupancy \\
\hline $\mathrm{Zn1}$ & 1 & 1.28933 & 0.56556 & 0.5 \\
\hline $\mathrm{Zn} 2$ & 0.934 & 1.21215 & 0.5 & 0.5 \\
\hline $\mathrm{O} 1$ & 1 & 1.25057 & 0.5 & 0.25 \\
\hline $\mathrm{O} 2$ & 0.88818 & 1.2235 & 0.55917 & 1 \\
\hline $\mathrm{O} 3$ & 0.94057 & 1.28227 & 0.61005 & 1 \\
\hline $\mathrm{O} 4$ & 1 & 1.35791 & 0.54754 & 0.5 \\
\hline $\mathrm{O} 5$ & 0.95216 & 1.14275 & 0.5 & 0.5 \\
\hline $\mathrm{C} 1$ & 0.89504 & 1.25506 & 0.60228 & 1 \\
\hline $\mathrm{C} 2$ & 0.84909 & 1.26377 & 0.64275 & 1 \\
\hline $\mathrm{H} 2$ & 0.86056 & 1.27794 & 0.68533 & 1 \\
\hline C3 & 0.79455 & 1.26225 & 0.62651 & 1 \\
\hline $\mathrm{H} 3$ & 0.78266 & 1.24718 & 0.58436 & 1 \\
\hline $\mathrm{C} 4$ & 0.74975 & 1.27881 & 0.66672 & 1 \\
\hline $\mathrm{C} 5$ & 0.70322 & 1.24257 & 0.67434 & 1 \\
\hline H5 & 0.69819 & 1.20581 & 0.6466 & 1 \\
\hline C6 & 1 & 1.38278 & 0.5 & 0.25 \\
\hline C7 & 1 & 1.44243 & 0.5 & 0.25 \\
\hline $\mathrm{C} 8$ & 1 & 1.47105 & 0.55228 & 0.5 \\
\hline H8 & 1 & 1.449 & 0.593 & 0.5 \\
\hline C9 & 1 & 1.11782 & 0.5 & 0.25 \\
\hline $\mathrm{C} 10$ & 1 & 1.05782 & 0.5 & 0.25 \\
\hline $\mathrm{C} 11$ & 0.94777 & 1.02904 & 0.5 & 0.5 \\
\hline H11 & 0.90706 & 1.05109 & 0.5 & 0.5 \\
\hline
\end{tabular}


Table S3. Crystallographic information and atomic fractional coordinates for the structure model of MOF-905- $\mathrm{Me}_{2}$.

\begin{tabular}{|c|c|c|c|c|}
\hline Identification Code & \multicolumn{4}{|c|}{ MOF-905-Me $\mathrm{Me}_{2}$} \\
\hline Empirical Formula & \multicolumn{4}{|c|}{$\mathrm{C}_{30} \mathrm{H}_{18} \mathrm{O}_{13} \mathrm{Zn}_{4}$} \\
\hline Formula Weight & \multicolumn{4}{|c|}{848} \\
\hline Crystal System & \multicolumn{4}{|l|}{ Cubic } \\
\hline Space Group & \multicolumn{4}{|l|}{$P m \overline{3}$} \\
\hline$a / \AA$ & \multicolumn{4}{|l|}{24.7215} \\
\hline$V / \AA^{3}$ & \multicolumn{4}{|l|}{15108} \\
\hline$Z$ & \multicolumn{4}{|l|}{6} \\
\hline$d / \mathrm{g} \mathrm{cm}^{-3}$ & \multicolumn{4}{|l|}{0.559} \\
\hline Atoms & $x$ & $y$ & $Z$ & Occupancy \\
\hline Zn1 & 0.43944 & 0 & 0.70921 & 0.5 \\
\hline $\mathrm{Zn} 2$ & 0.5 & 0.06391 & 0.78812 & 0.5 \\
\hline $\mathrm{O} 1$ & 0.5 & 0 & 0.74952 & 0.25 \\
\hline $\mathrm{O} 2$ & 0.39683 & 0.05663 & 0.71804 & 1 \\
\hline $\mathrm{O} 3$ & 0.44014 & 0.10745 & 0.77705 & 1 \\
\hline $\mathrm{O} 4$ & 0.45569 & 0 & 0.64147 & 0.5 \\
\hline $\mathrm{O} 5$ & 0.5 & 0.04493 & 0.85697 & 0.5 \\
\hline $\mathrm{C} 1$ & 0.3997 & 0.09914 & 0.74614 & 1 \\
\hline $\mathrm{C} 2$ & 0.35379 & 0.13741 & 0.74062 & 1 \\
\hline $\mathrm{H} 2$ & 0.31894 & 0.12148 & 0.7195 & 1 \\
\hline $\mathrm{C} 3$ & 0.35283 & 0.18893 & 0.75768 & 1 \\
\hline H3 & 0.38683 & 0.20428 & 0.78069 & 1 \\
\hline $\mathrm{C} 4$ & 0.30573 & 0.22584 & 0.74719 & 1 \\
\hline $\mathrm{C} 5$ & 0.29705 & 0.2702 & 0.78177 & 1 \\
\hline H5 & 0.32552 & 0.27699 & 0.81429 & 1 \\
\hline C6 & 0.5 & 0 & 0.61641 & 0.25 \\
\hline $\mathrm{C} 7$ & 0.5 & 0 & 0.5569 & 0.25 \\
\hline $\mathrm{C} 8$ & 0.45036 & 0 & 0.52814 & 0.5 \\
\hline C9 & 0.39618 & 0 & 0.55617 & 0.25 \\
\hline H9a & 0.37627 & 0.03171 & 0.54586 & 0.25 \\
\hline $\mathrm{H} 9 \mathrm{~b}$ & 0.37627 & -0.03171 & 0.54586 & 0.25 \\
\hline H9c & 0.40151 & 0 & 0.59464 & 0.25 \\
\hline $\mathrm{C} 10$ & 0.5 & 0 & 0.88212 & 0.25 \\
\hline C11 & 0.5 & 0 & 0.94245 & 0.25 \\
\hline $\mathrm{C} 12$ & 0.5 & -0.04947 & 0.97167 & 0.5 \\
\hline C13 & 0.5 & -0.10357 & 0.94364 & 0.25 \\
\hline $\mathrm{H} 13 \mathrm{a}$ & 0.53171 & -0.12348 & 0.95394 & 0.25 \\
\hline $\mathrm{H} 13 \mathrm{~b}$ & 0.46829 & -0.12348 & 0.95394 & 0.25 \\
\hline $\mathrm{H} 13 \mathrm{c}$ & 0.5 & -0.09822 & 0.90518 & 0.25 \\
\hline
\end{tabular}


Table S4. Crystallographic information and atomic fractional coordinates for the structure model of MOF-905-Naph.

\begin{tabular}{|c|c|c|c|c|}
\hline Identification Code & \multicolumn{4}{|c|}{ MOF-905-Naph } \\
\hline Empirical Formula & \multicolumn{4}{|c|}{$\mathrm{C}_{32} \mathrm{H}_{16} \mathrm{O}_{13} \mathrm{Zn}_{4}$} \\
\hline Formula Weight & \multicolumn{4}{|c|}{870.01} \\
\hline Crystal System & \multicolumn{4}{|l|}{ Cubic } \\
\hline Space Group & \multicolumn{4}{|l|}{$P m \overline{3}$} \\
\hline$a / \AA$ & \multicolumn{4}{|l|}{24.74010} \\
\hline$V / \AA^{3}$ & \multicolumn{4}{|l|}{15142.7} \\
\hline$Z$ & \multicolumn{4}{|l|}{6} \\
\hline$d / \mathrm{g} \mathrm{cm}^{-3}$ & \multicolumn{4}{|l|}{0.572} \\
\hline Atoms & $x$ & $y$ & $z$ & Occupancy \\
\hline Zn1 & 0.43951 & 0 & 0.71012 & 0.5 \\
\hline $\mathrm{Zn} 2$ & 0.5 & 0.06385 & 0.787 & 0.5 \\
\hline $\mathrm{O} 1$ & 0.5 & 0 & 0.74942 & 0.25 \\
\hline $\mathrm{O} 2$ & 0.39676 & 0.05657 & 0.71816 & 1 \\
\hline $\mathrm{O} 3$ & 0.4402 & 0.10754 & 0.77663 & 1 \\
\hline $\mathrm{O} 4$ & 0.45681 & 0 & 0.64366 & 0.5 \\
\hline $\mathrm{O} 5$ & 0.5 & 0.04382 & 0.85458 & 0.5 \\
\hline $\mathrm{C} 1$ & 0.39968 & 0.09919 & 0.74596 & 1 \\
\hline $\mathrm{C} 2$ & 0.35381 & 0.13745 & 0.74037 & 1 \\
\hline $\mathrm{H} 2$ & 0.31905 & 0.12156 & 0.71915 & 1 \\
\hline $\mathrm{C} 3$ & 0.35275 & 0.18889 & 0.75759 & 1 \\
\hline $\mathrm{H} 3$ & 0.38666 & 0.20417 & 0.78071 & 1 \\
\hline $\mathrm{C} 4$ & 0.30566 & 0.22579 & 0.74716 & 1 \\
\hline $\mathrm{C} 5$ & 0.29702 & 0.27011 & 0.78176 & 1 \\
\hline H5 & 0.3255 & 0.27685 & 0.81424 & 1 \\
\hline C6 & 0.5 & 0 & 0.61795 & 0.25 \\
\hline $\mathrm{C} 7$ & 0.5 & 0 & 0.55768 & 0.25 \\
\hline $\mathrm{C} 8$ & 0.44908 & 0 & 0.52875 & 0.5 \\
\hline C9 & 0.39673 & 0 & 0.55497 & 0.25 \\
\hline H9 & 0.38718 & 0 & 0.59678 & 0.25 \\
\hline $\mathrm{C} 10$ & 0.34828 & 0 & 0.52742 & 0.25 \\
\hline H10 & 0.31013 & 0 & 0.54903 & 0.25 \\
\hline $\mathrm{C} 11$ & 0.5 & 0 & 0.88042 & 0.25 \\
\hline C12 & 0.5 & 0 & 0.9416 & 0.25 \\
\hline $\mathrm{C} 13$ & 0.5 & 0.0507 & 0.97107 & 0.5 \\
\hline $\mathrm{C} 14$ & 0.5 & 0.10299 & 0.94495 & 0.25 \\
\hline H14 & 0.5 & 0.11237 & 0.90316 & 0.25 \\
\hline $\mathrm{C} 15$ & 0.5 & 0.15141 & 0.97257 & 0.25 \\
\hline H15 & 0.5 & 0.18956 & 0.95096 & 0.25 \\
\hline
\end{tabular}


Table S5. Crystallographic information and atomic fractional coordinates for the structure model of MOF-905- $\mathrm{NO}_{2}$.

\begin{tabular}{|c|c|c|c|c|}
\hline Identification Code & \multicolumn{4}{|c|}{ MOF-905-NO $\mathrm{NO}_{2}$} \\
\hline Empirical Formula & \multicolumn{4}{|c|}{$\mathrm{C}_{28} \mathrm{H}_{12} \mathrm{NO}_{15} \mathrm{Zn}_{4}$} \\
\hline Formula Weight & \multicolumn{4}{|c|}{863.95} \\
\hline Crystal System & \multicolumn{4}{|l|}{ Cubic } \\
\hline Space Group & \multicolumn{4}{|l|}{$P m \overline{3}$} \\
\hline$a / \AA$ & \multicolumn{4}{|l|}{23.99180} \\
\hline$V / \AA^{3}$ & \multicolumn{4}{|l|}{13809.8} \\
\hline$Z$ & \multicolumn{4}{|l|}{6} \\
\hline$d / \mathrm{g} \mathrm{cm}^{-3}$ & \multicolumn{4}{|l|}{0.623} \\
\hline Atoms & $x$ & $y$ & $z$ & Occupancy \\
\hline Zn1 & 0.43694 & 0 & 0.70981 & 0.5 \\
\hline $\mathrm{Zn} 2$ & 0.5 & 0.06494 & 0.78691 & 0.5 \\
\hline O1 & 0.5 & 0 & 0.74889 & 0.25 \\
\hline $\mathrm{O} 2$ & 0.3933 & 0.05766 & 0.71761 & 1 \\
\hline $\mathrm{O} 3$ & 0.44078 & 0.10959 & 0.77619 & 1 \\
\hline $\mathrm{O} 4$ & 0.45418 & 0 & 0.64224 & 0.5 \\
\hline $\mathrm{O} 5$ & 0.5 & 0.04629 & 0.85542 & 0.5 \\
\hline O6 & 0.54687 & -0.12971 & 0.93321 & 0.25 \\
\hline $\mathrm{O} 7$ & 0.37042 & 0.04689 & 0.56631 & 0.25 \\
\hline N1 & 0.39579 & 0 & 0.55641 & 0.125 \\
\hline N2 & 0.5 & -0.10405 & 0.94237 & 0.125 \\
\hline $\mathrm{C} 1$ & 0.39874 & 0.10173 & 0.74533 & 1 \\
\hline $\mathrm{C} 2$ & 0.35524 & 0.14363 & 0.73905 & 1 \\
\hline $\mathrm{H} 2$ & 0.31564 & 0.12927 & 0.72275 & 1 \\
\hline $\mathrm{C} 3$ & 0.36389 & 0.19789 & 0.74727 & 1 \\
\hline H3 & 0.40277 & 0.21235 & 0.76515 & 1 \\
\hline $\mathrm{C} 4$ & 0.32005 & 0.23872 & 0.73382 & 1 \\
\hline $\mathrm{C} 5$ & 0.31189 & 0.28422 & 0.76914 & 1 \\
\hline H5 & 0.34012 & 0.29042 & 0.80384 & 1 \\
\hline C6 & 0.5 & 0 & 0.61718 & 0.25 \\
\hline $\mathrm{C} 7$ & 0.5 & 0 & 0.55747 & 0.25 \\
\hline $\mathrm{C} 8$ & 0.44874 & 0 & 0.52856 & 0.5 \\
\hline C9 & 0.5 & 0 & 0.88053 & 0.25 \\
\hline C10 & 0.5 & 0 & 0.94076 & 0.25 \\
\hline $\mathrm{C} 11$ & 0.5 & -0.05102 & 0.96986 & 0.5 \\
\hline
\end{tabular}




\section{Section S3 Powder X-ray Diffraction Patterns}

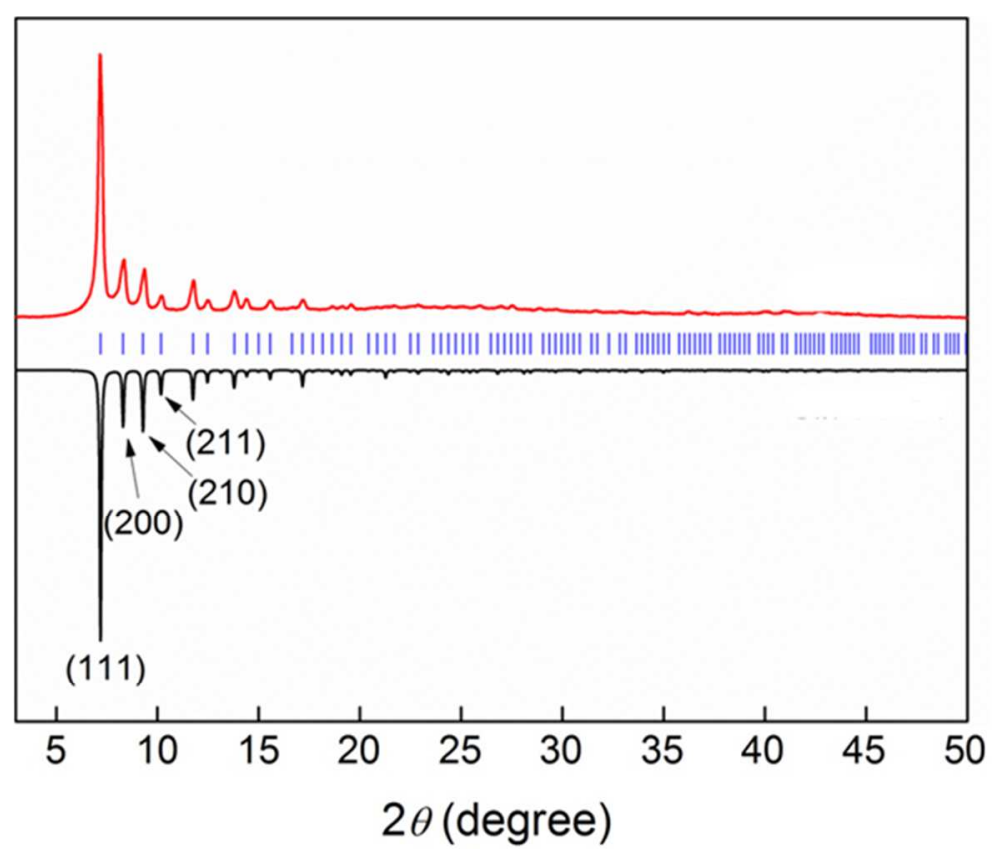

Figure S2. Comparison of the experimental PXRD patterns of MOF-950: activated (red) and the simulated pattern (black) from single crystal X-ray data. $(\lambda=1.54184 \AA$ )

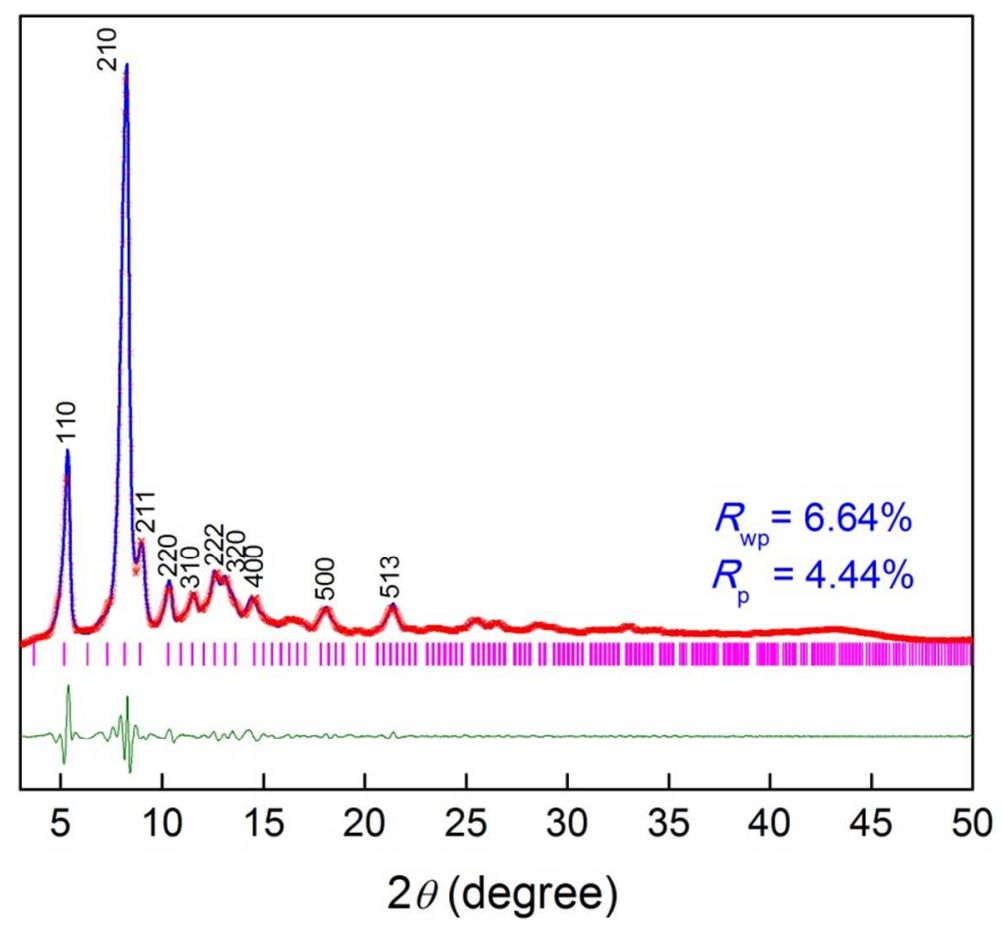

Figure S3. PXRD analysis of the activated sample of MOF-905 (red), the Pawley fitting (blue) from the modeled structure, and their difference (green). $(\lambda=1.54184 \AA)$ 


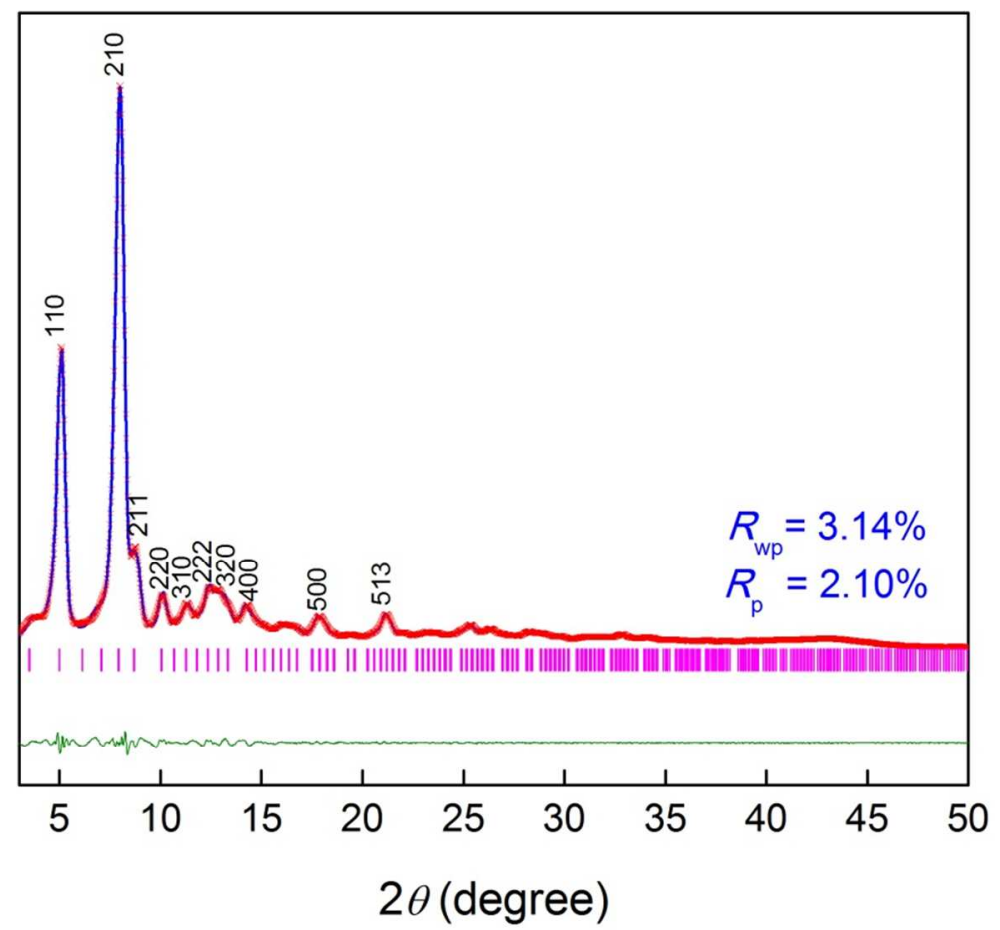

Figure S4. PXRD analysis of the activated sample of MOF-905-Me 2 (red), the Pawley fitting (blue) from the modeled structure, and their difference (green). $(\lambda=1.54184 \AA$ )

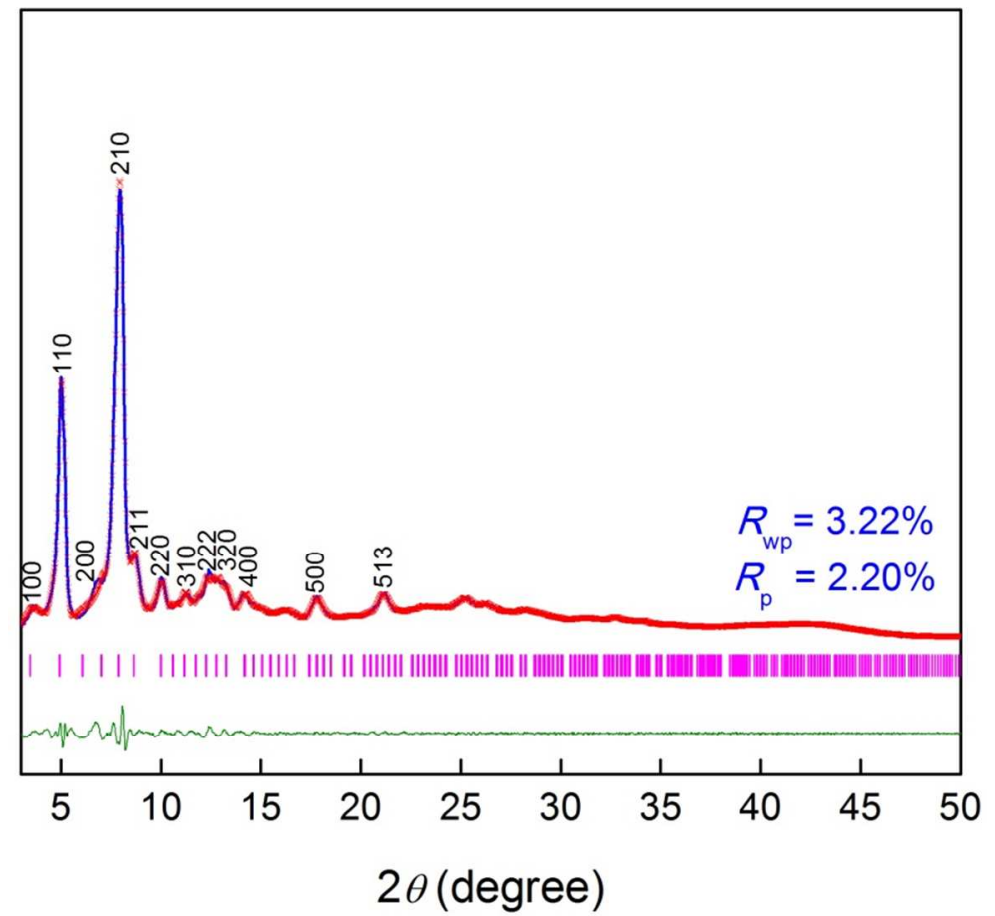

Figure S5. PXRD analysis of the activated sample of MOF-905-Naph (red), the Pawley fitting (blue) from the modeled structure, and their difference (green). $(\lambda=1.54184 \AA$ ) 


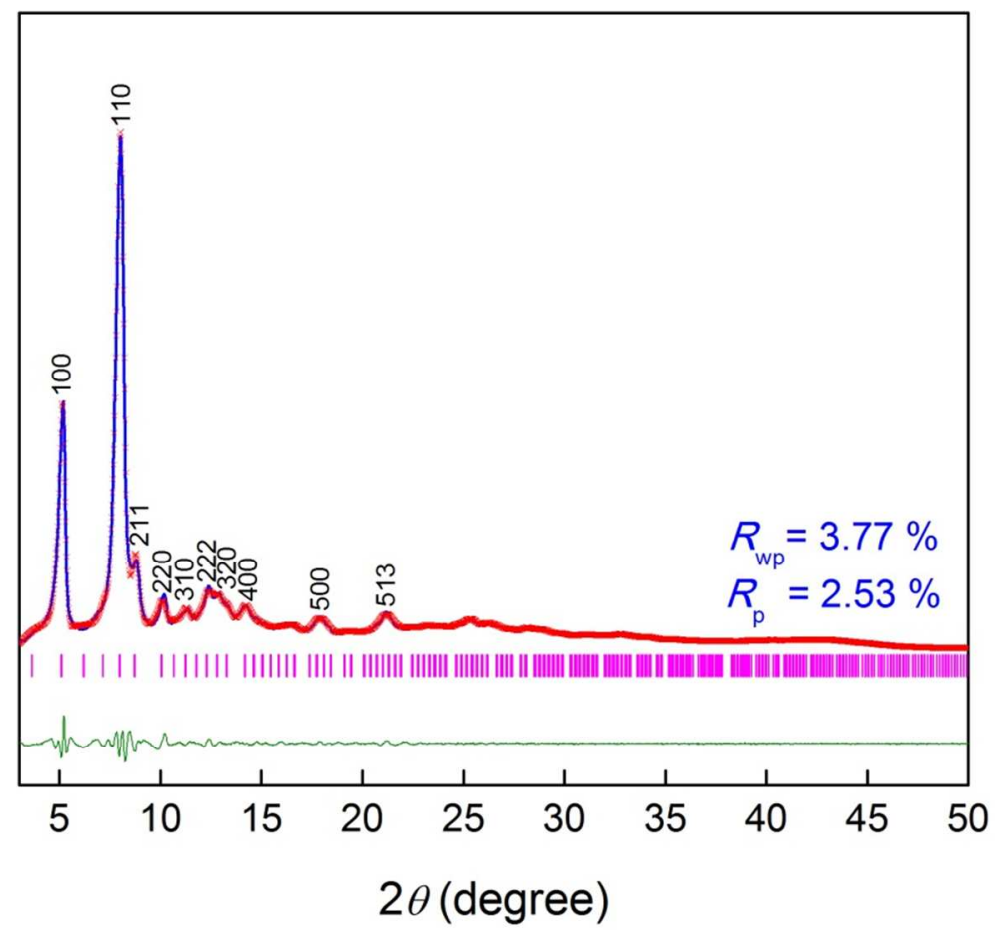

Figure S6. PXRD analysis of the activated sample of MOF-905- $\mathrm{NO}_{2}$ (red), the Pawley fitting (blue) from the modeled structure, and their difference (green). $(\lambda=1.54184 \AA)$ 


\section{Section S4 Thermal Gravimetric Analyses}

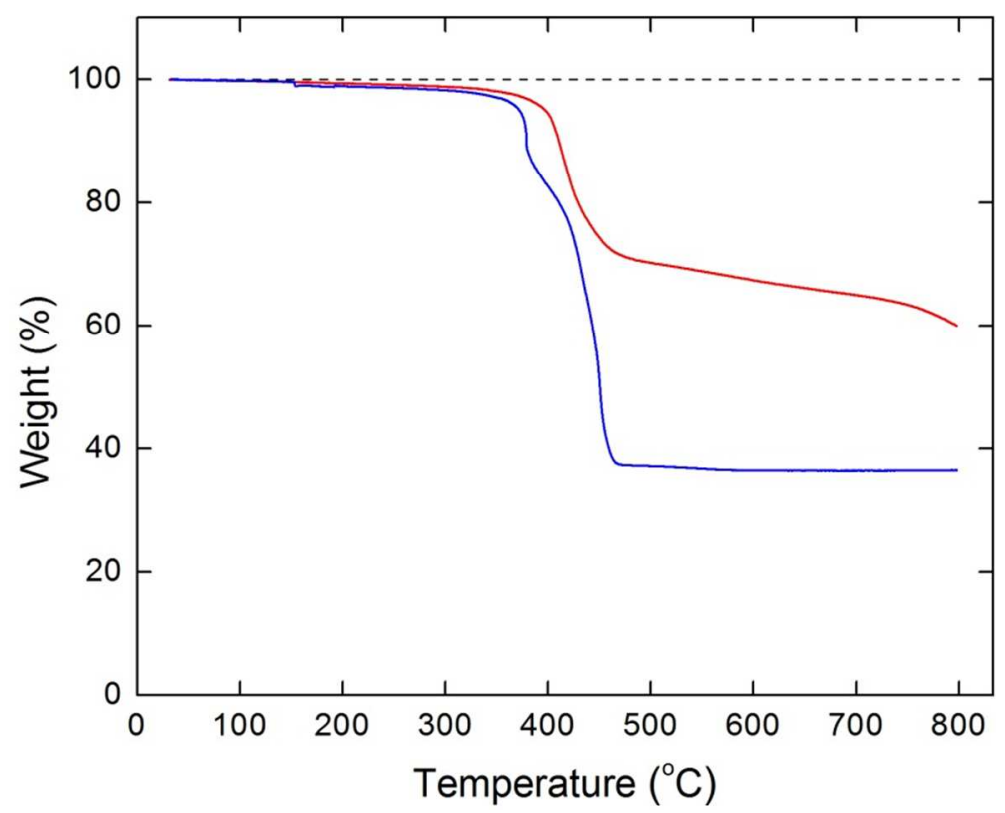

Figure S7. TGA traces for activated MOF-950, heating rate: $5^{\circ} \mathrm{C} \min ^{-1}$ in air (blue) and in $\mathrm{N}_{2}$ (red).

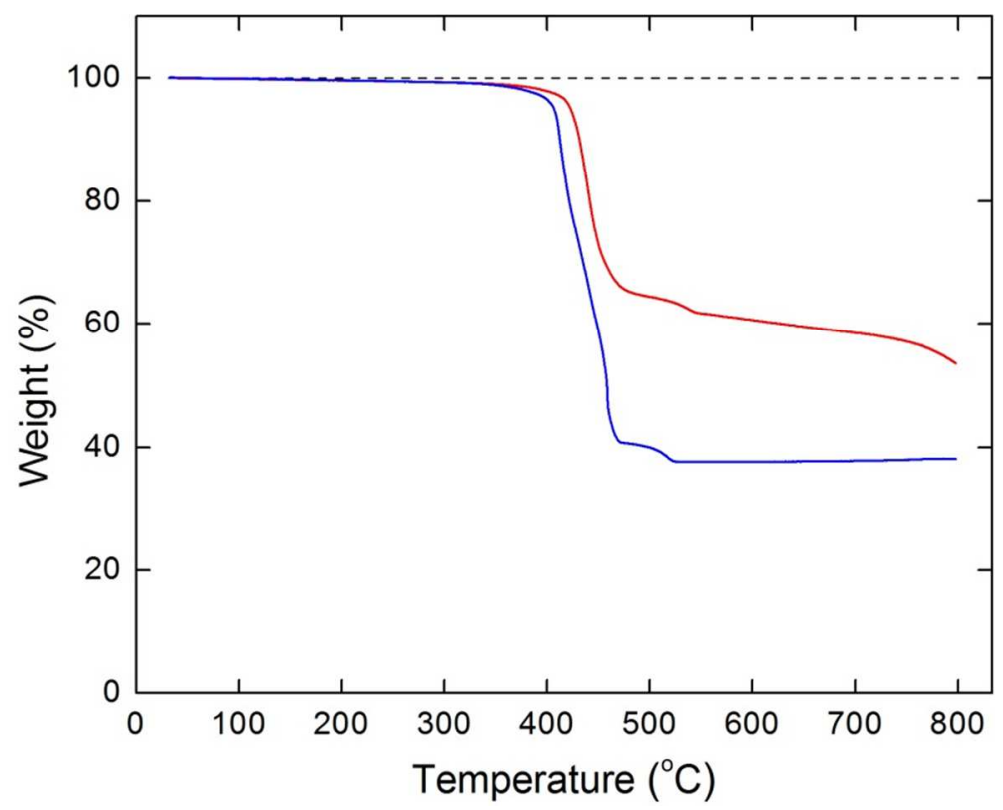

Figure S8. TGA traces for activated MOF-905, heating rate: $5^{\circ} \mathrm{C} \mathrm{min}^{-1}$ in air (blue) and in $\mathrm{N}_{2}$ (red). 


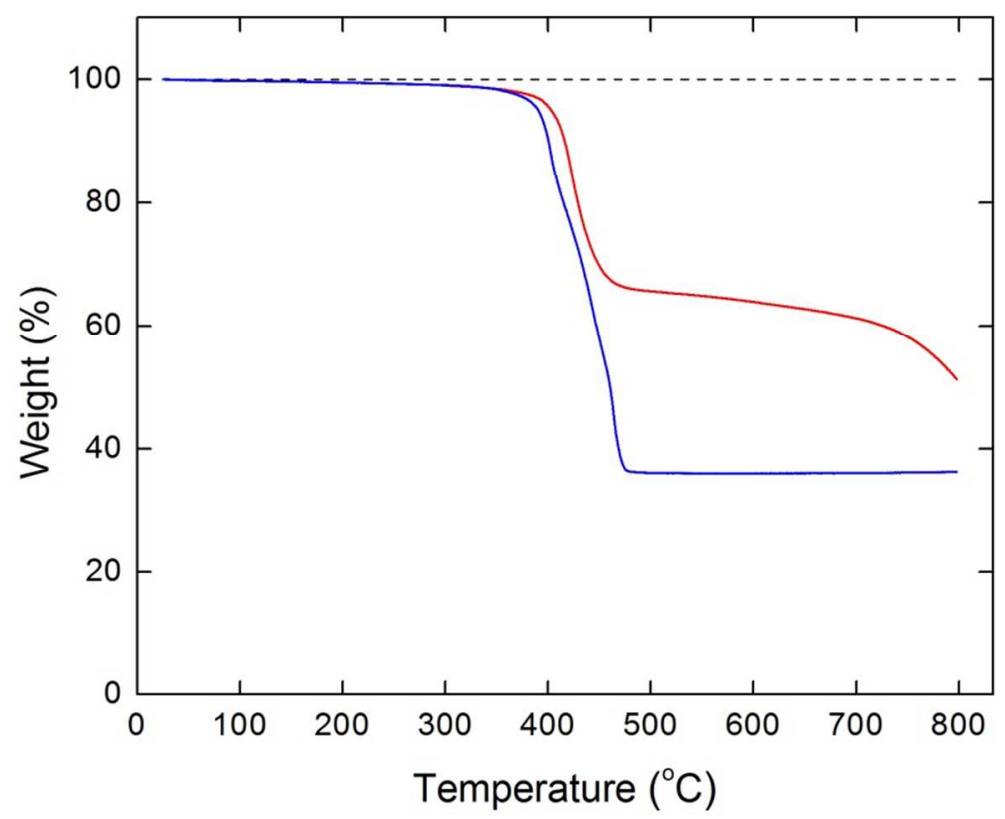

Figure S9. TGA traces for activated MOF-905-Me 2 , heating rate: $5{ }^{\circ} \mathrm{C} \mathrm{min}^{-1}$ in air (blue) and in $\mathrm{N}_{2}$ (red).

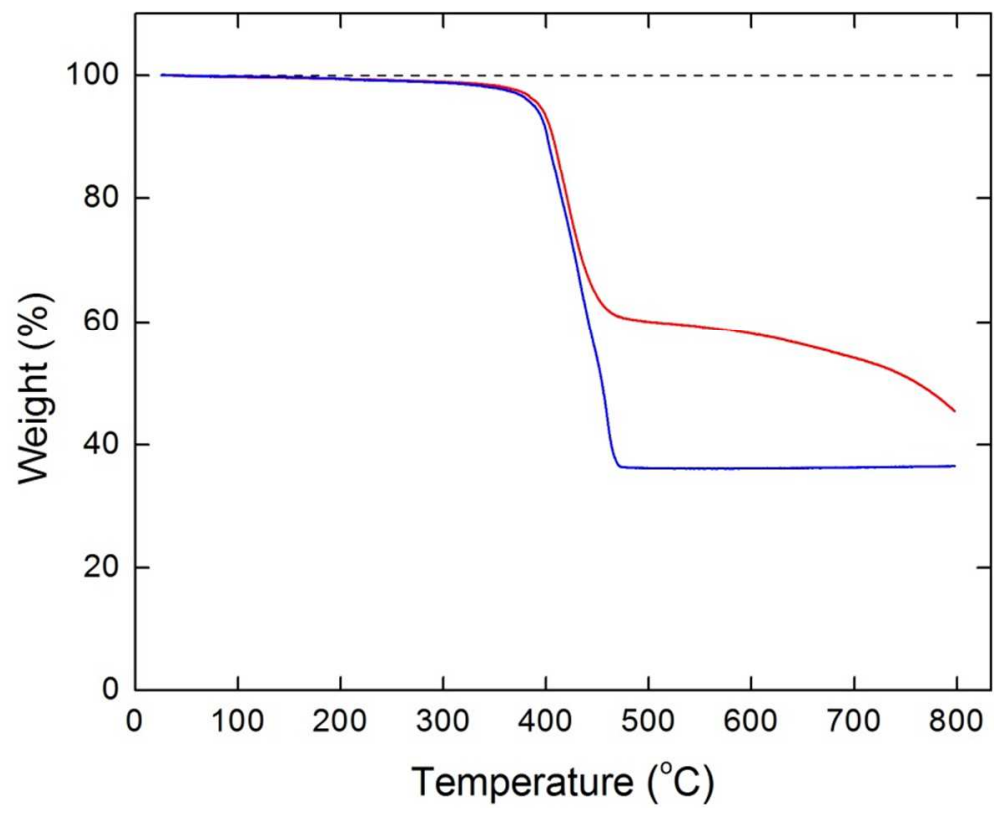

Figure S10. TGA traces for activated MOF-905-Naph, heating rate: $5{ }^{\circ} \mathrm{C} \mathrm{min}{ }^{-1}$ in air (blue) $\mathrm{N}_{2}$ (red). 


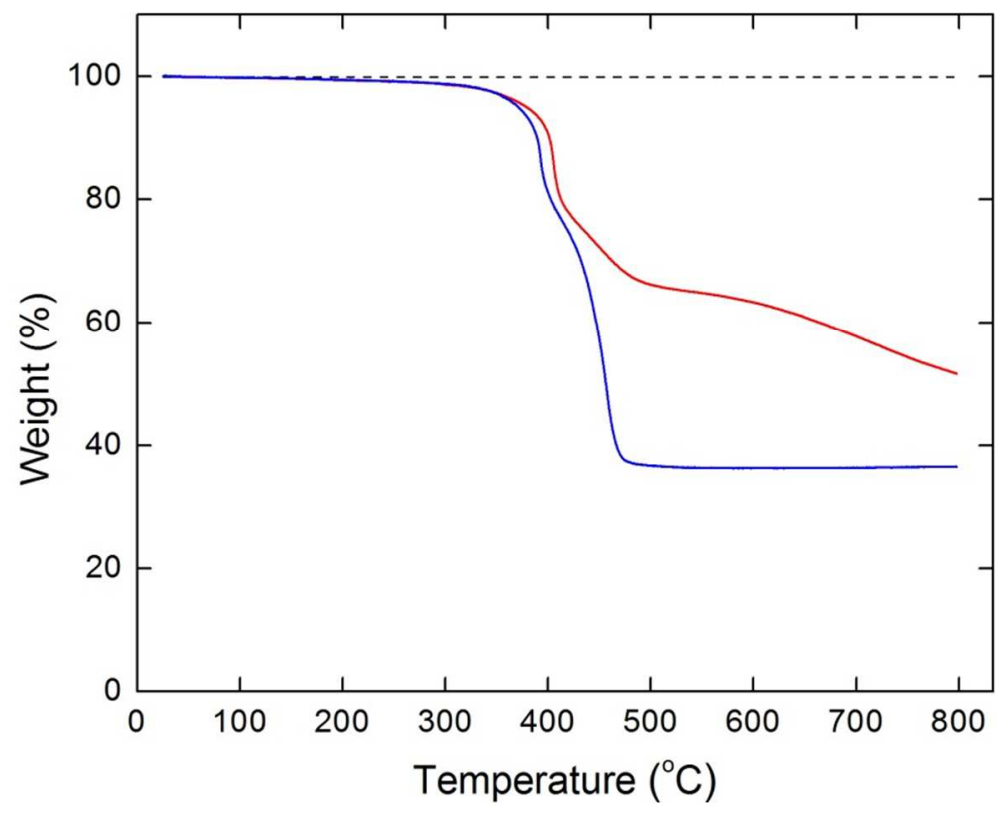

Figure S11. TGA traces for activated MOF-905- $\mathrm{NO}_{2}$, heating rate: $5{ }^{\circ} \mathrm{C} \mathrm{min}{ }^{-1}$ in air (blue) and in $\mathrm{N}_{2}$ (red). 
Section S5 $\mathrm{N}_{2}$ Adsorption Measurements

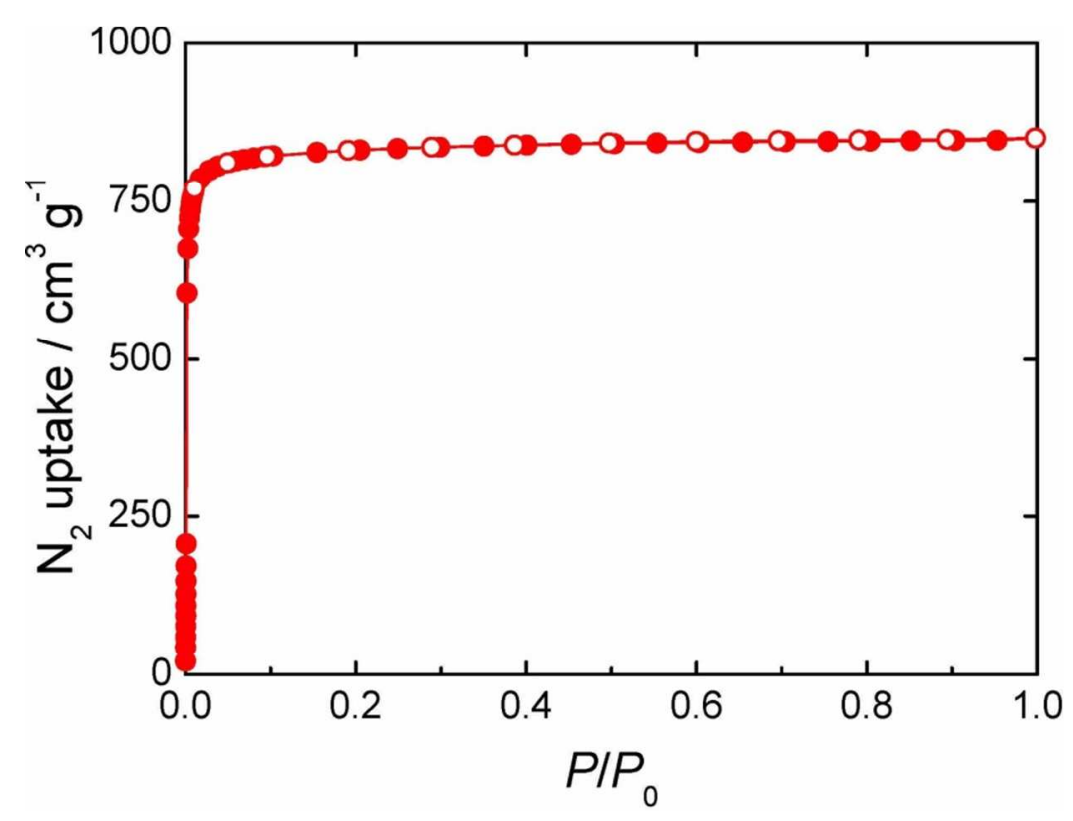

Figure S12. $\mathrm{N}_{2}$ adsorption isotherm of MOF-950 at $77 \mathrm{~K}$.

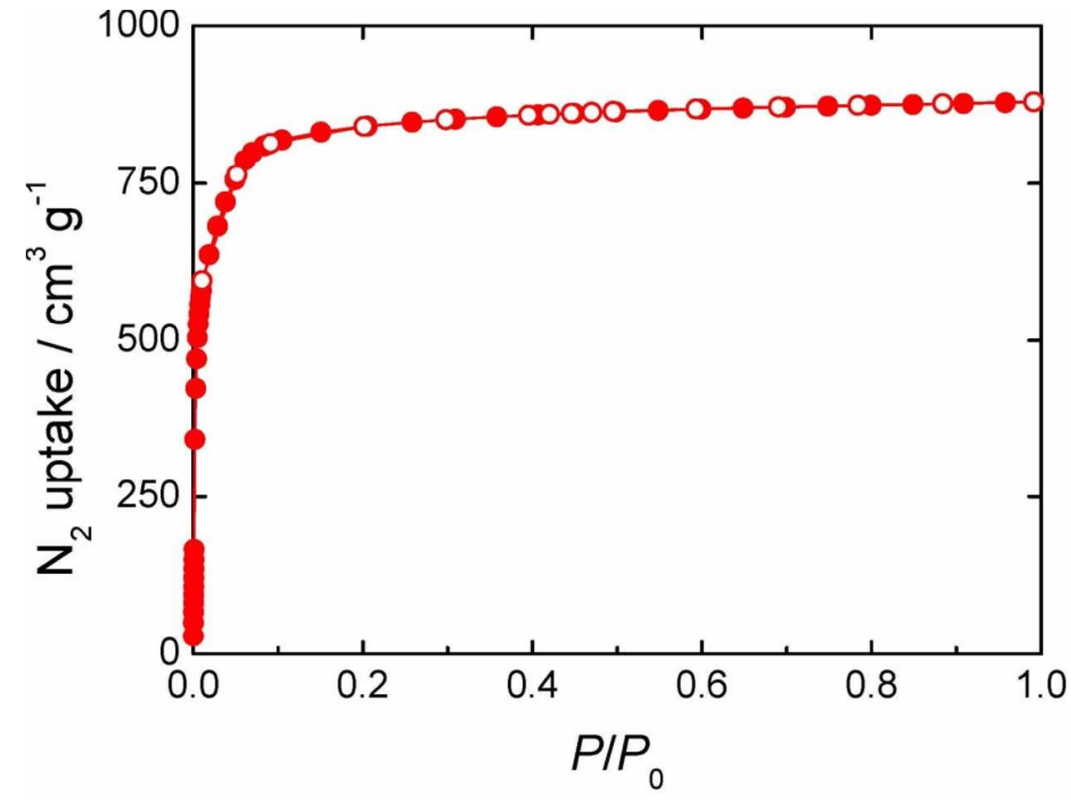

Figure S13. $\mathrm{N}_{2}$ adsorption isotherm of two sample batches of MOF-905 at $77 \mathrm{~K}$. 


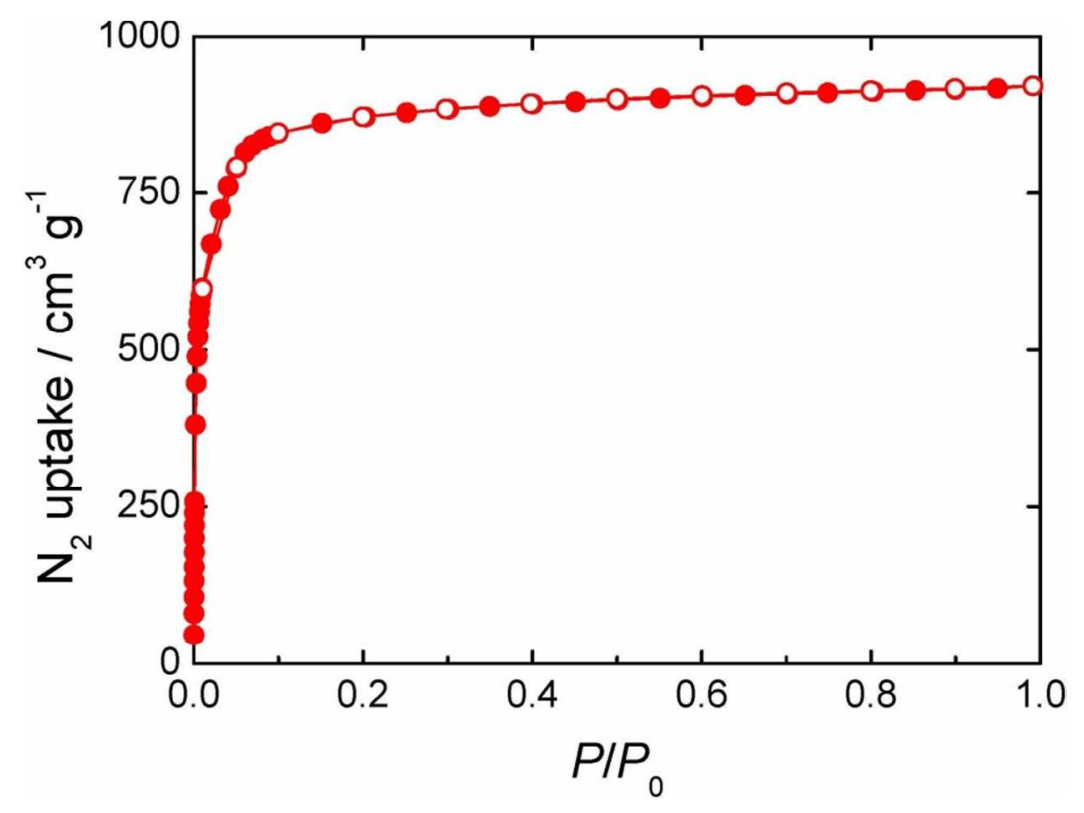

Figure S14. $\mathrm{N}_{2}$ adsorption isotherm of MOF-905- $\mathrm{Me}_{2}$ at $77 \mathrm{~K}$.

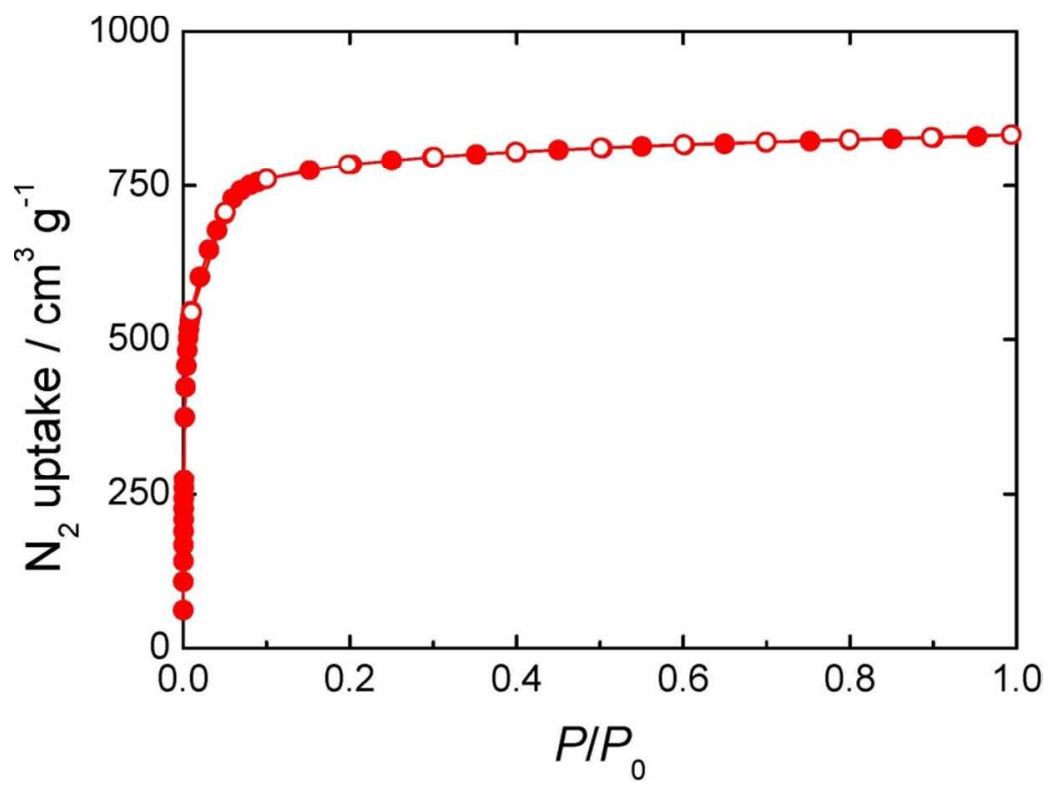

Figure S15. $\mathrm{N}_{2}$ adsorption isotherm of MOF-905-Naph at $77 \mathrm{~K}$. 


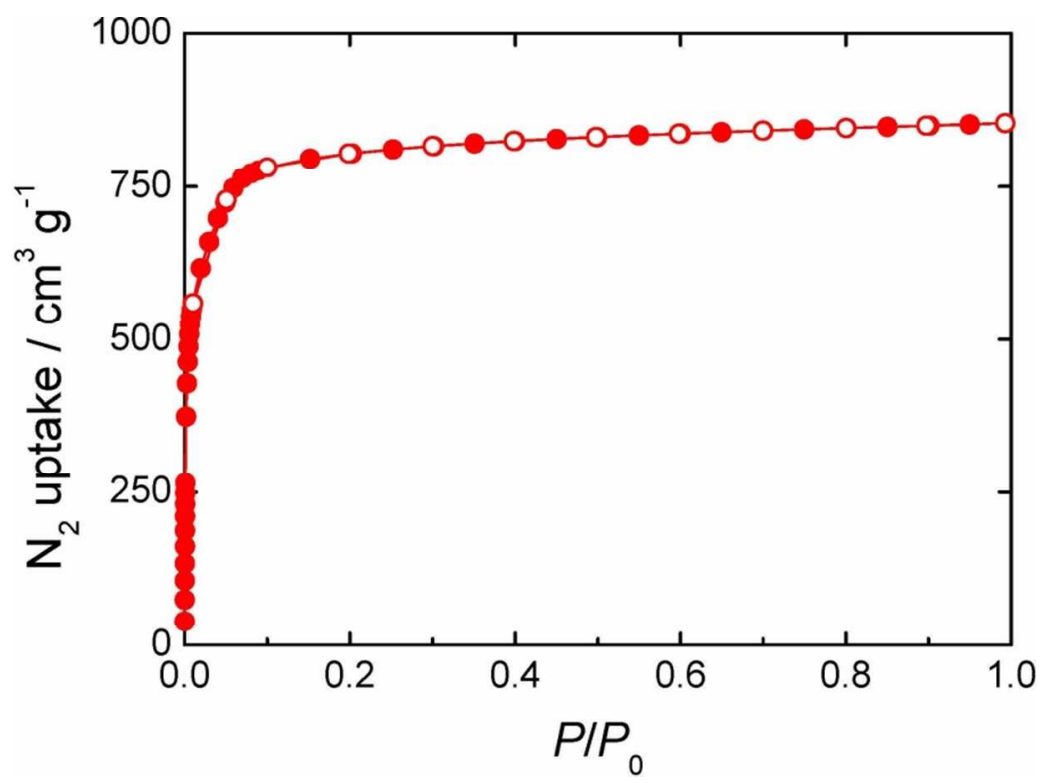

Figure S16. $\mathrm{N}_{2}$ adsorption isotherm of MOF-905- $\mathrm{NO}_{2}$ at $77 \mathrm{~K}$. 


\section{Section S6 Low Pressure $\mathrm{CH}_{4}$ Adsorption Measurements}

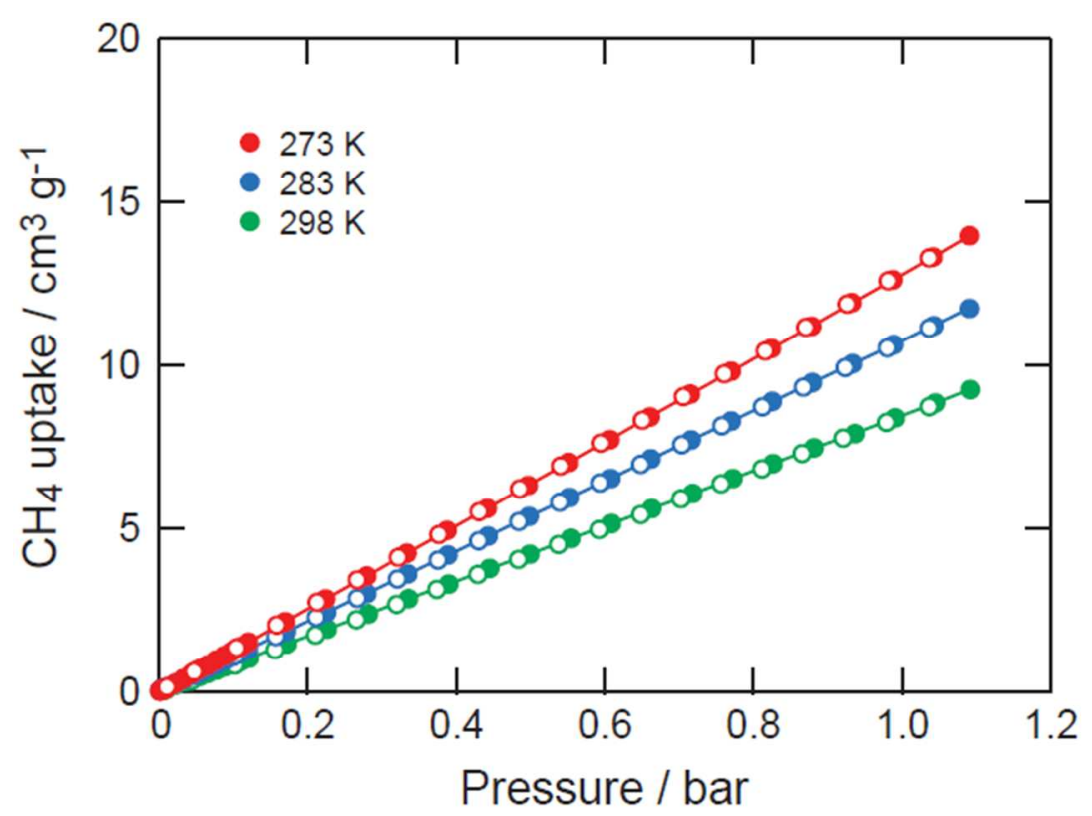

Figure S17. Low-pressure $\mathrm{CH}_{4}$ adsorption isotherms for MOF-950 measured at 273, 283, and $298 \mathrm{~K}$.

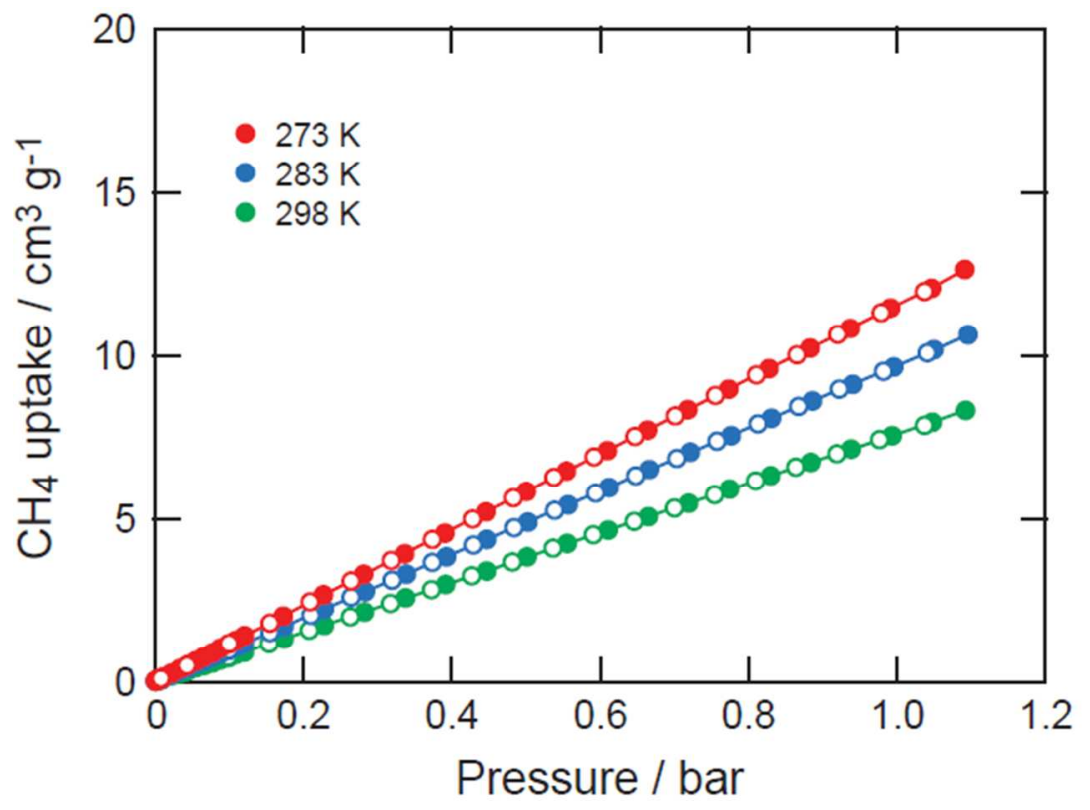

Figure S18. Low-pressure $\mathrm{CH}_{4}$ adsorption isotherms for MOF-905 measured at 273, 283, and $298 \mathrm{~K}$. 


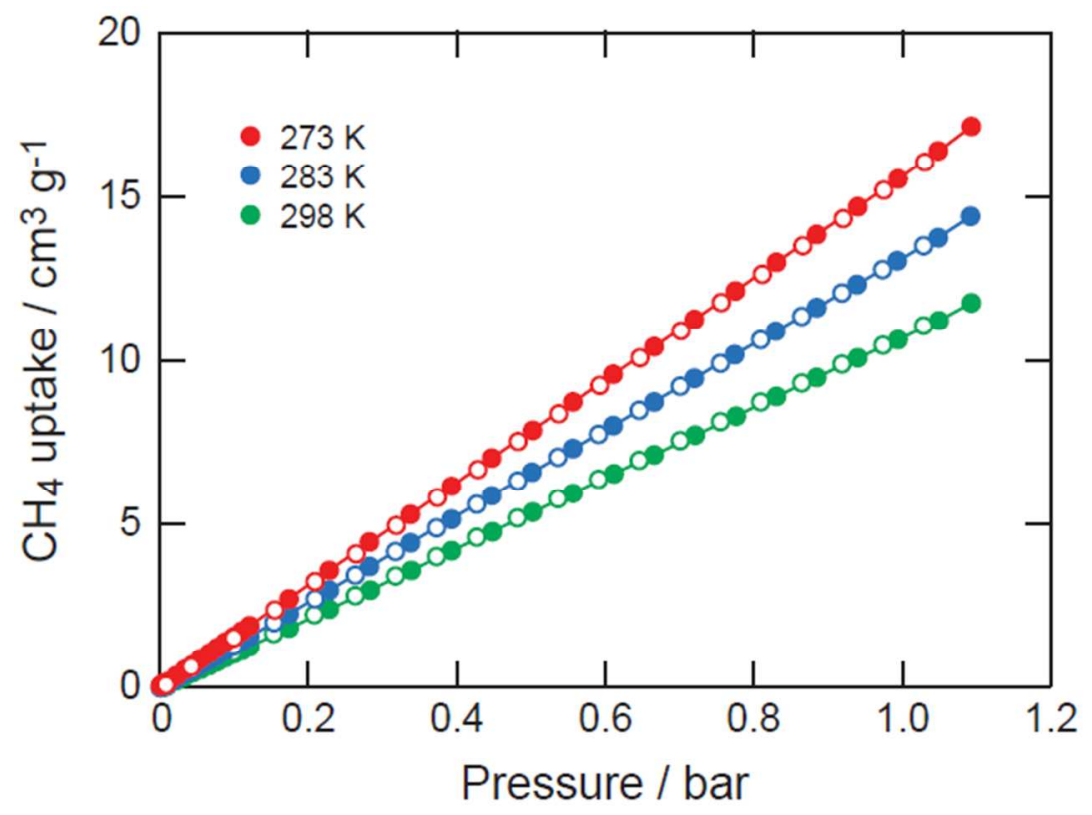

Figure S19. Low-pressure $\mathrm{CH}_{4}$ adsorption isotherms for MOF-905- $\mathrm{Me}_{2}$ measured at 273, 283, and $298 \mathrm{~K}$.

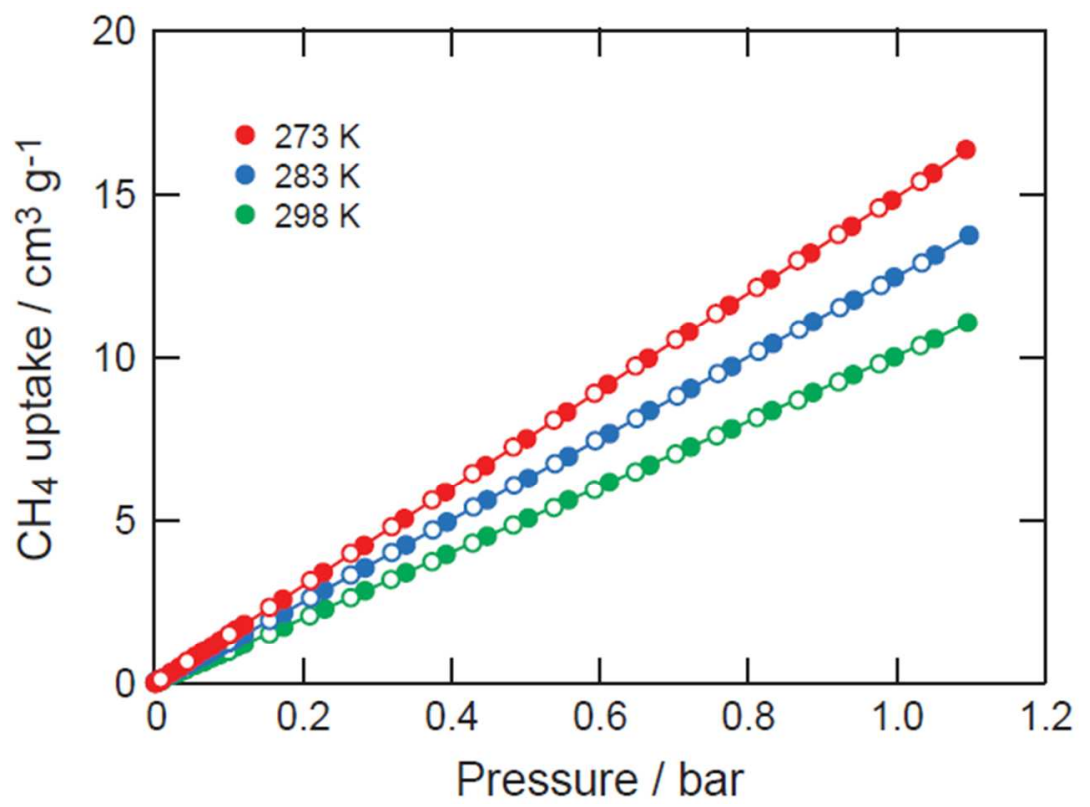

Figure S20. Low-pressure $\mathrm{CH}_{4}$ adsorption isotherms for MOF-905-Naph measured at 273, 283, and $298 \mathrm{~K}$. 


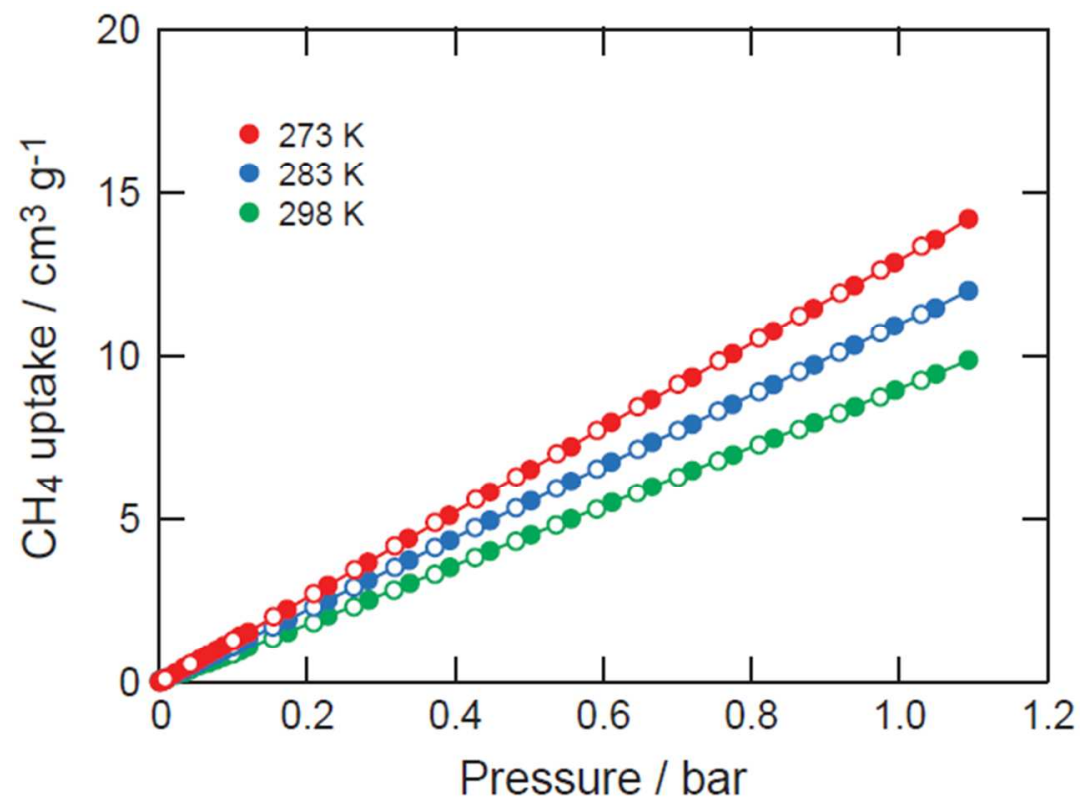

Figure S21. Low-pressure $\mathrm{CH}_{4}$ adsorption isotherms for MOF-905- $\mathrm{NO}_{2}$ measured at 273, 283, and $298 \mathrm{~K}$.

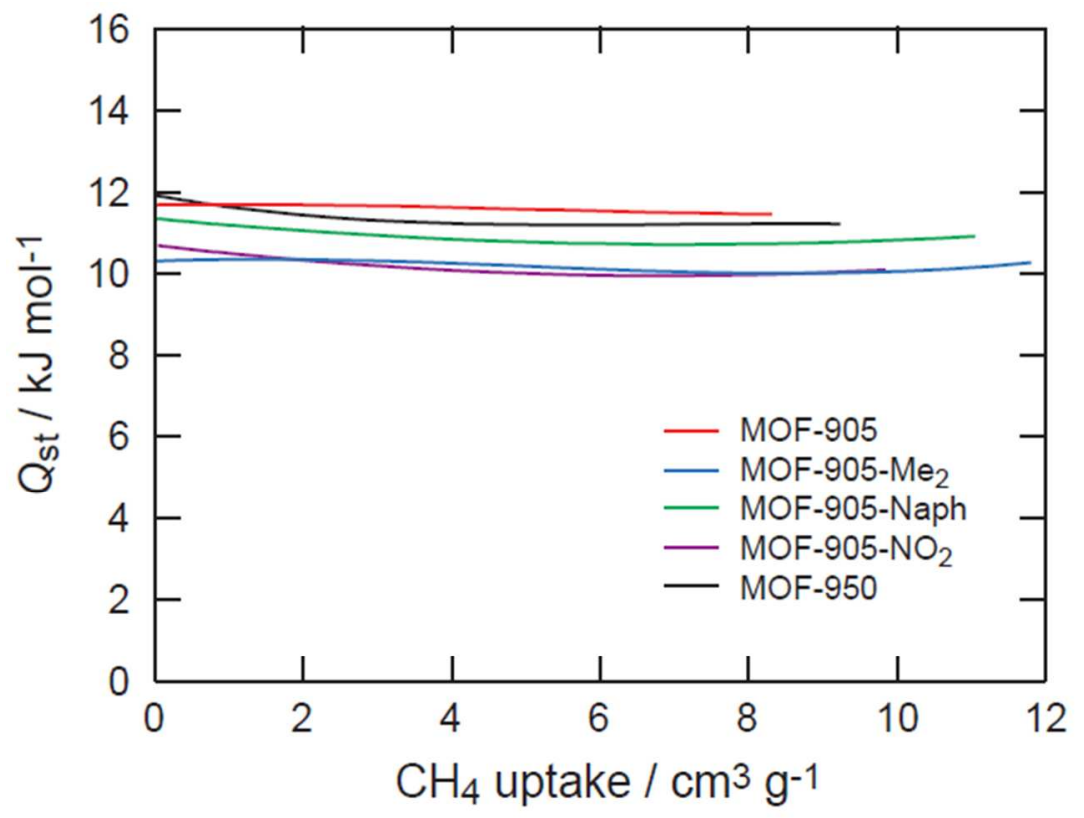

Figure S22. Isosteric enthalpies of adsorption for the MOFs investigated. 


\section{Section S7 High Pressure $\mathrm{CH}_{4}$ Adsorption Measurements}

\section{Excess and total $\mathrm{CH}_{4}$ isotherms}

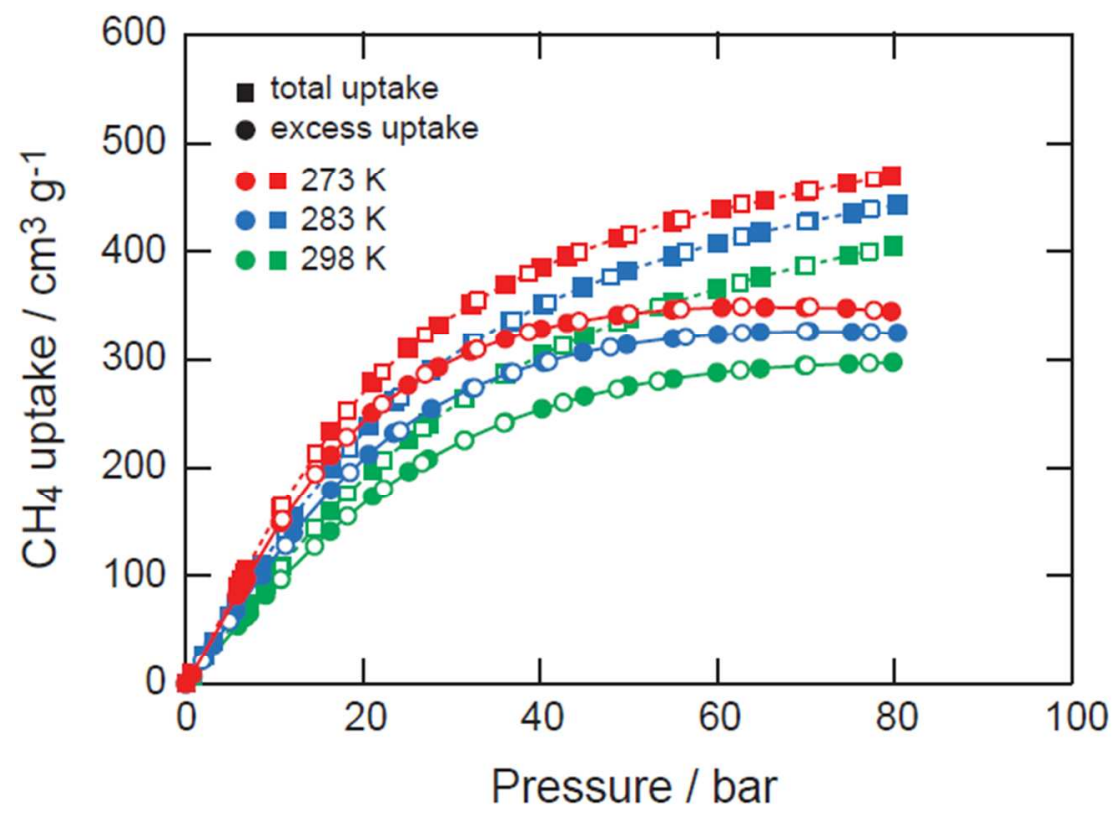

Figure S23. Excess and total $\mathrm{CH}_{4}$ adsorption isotherms for MOF-950 measured at 273, 283 , and $298 \mathrm{~K}$.

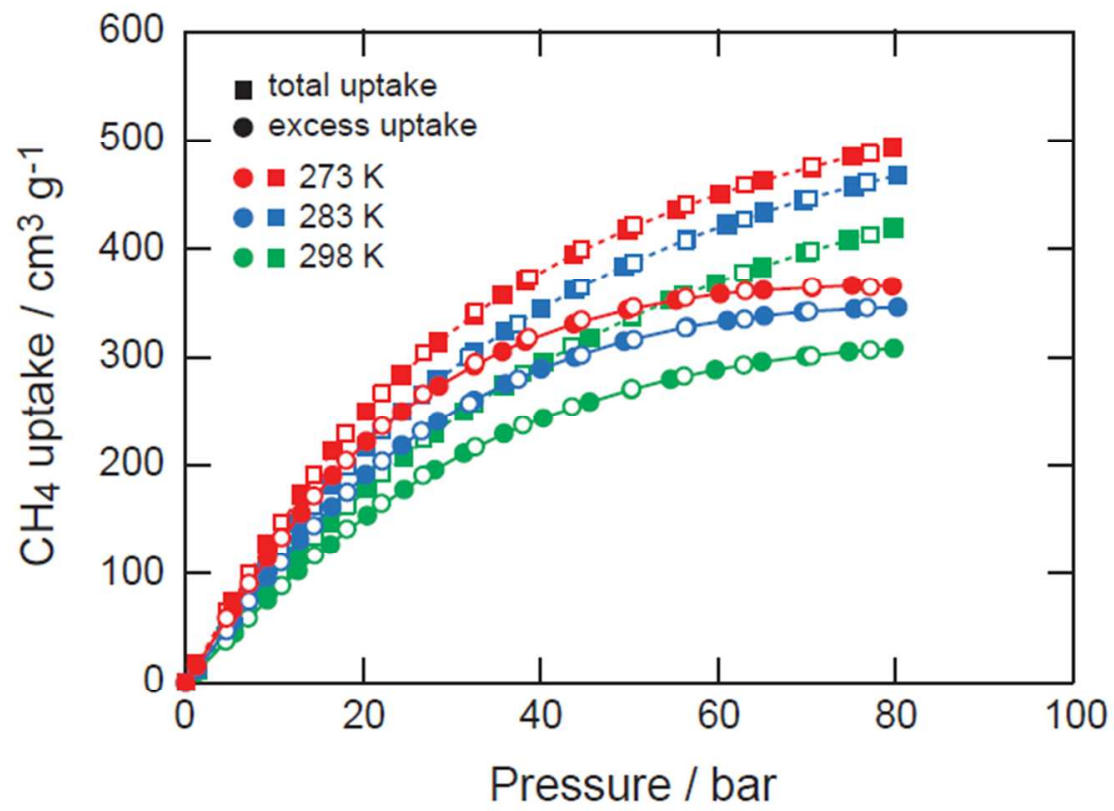

Figure S24. Excess and total $\mathrm{CH}_{4}$ adsorption isotherms for MOF-905 measured at 273, 283 , and $298 \mathrm{~K}$. 


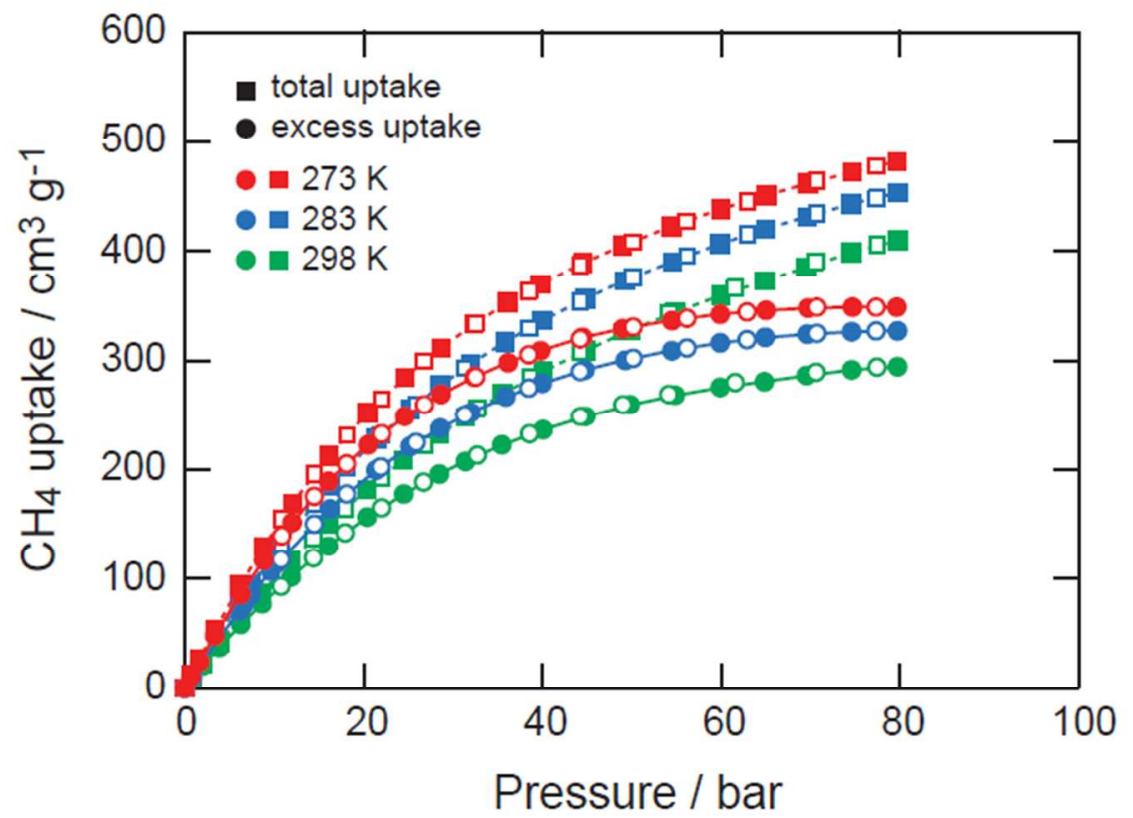

Figure S25. Excess and total $\mathrm{CH}_{4}$ adsorption isotherms for MOF-905- $\mathrm{Me}_{2}$ measured at 273, 283, and $298 \mathrm{~K}$.

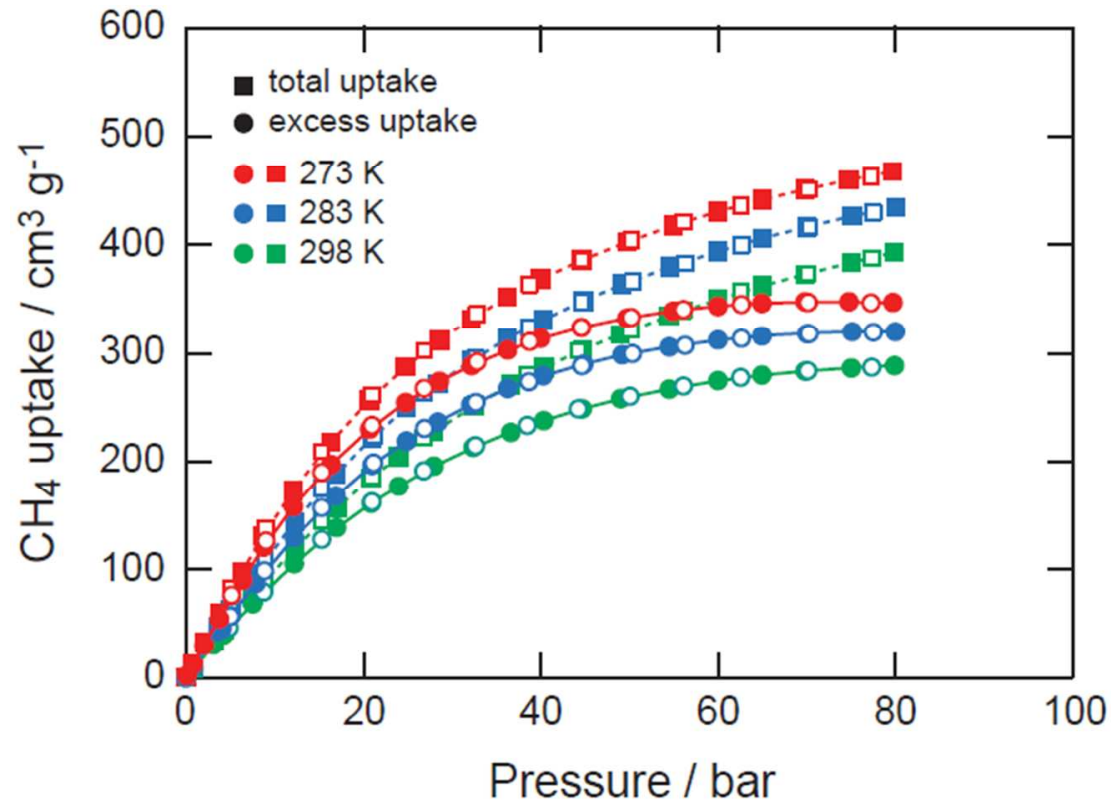

Figure S26. Excess and total $\mathrm{CH}_{4}$ adsorption isotherms for MOF-905-Naph measured at 273,283 , and $298 \mathrm{~K}$. 


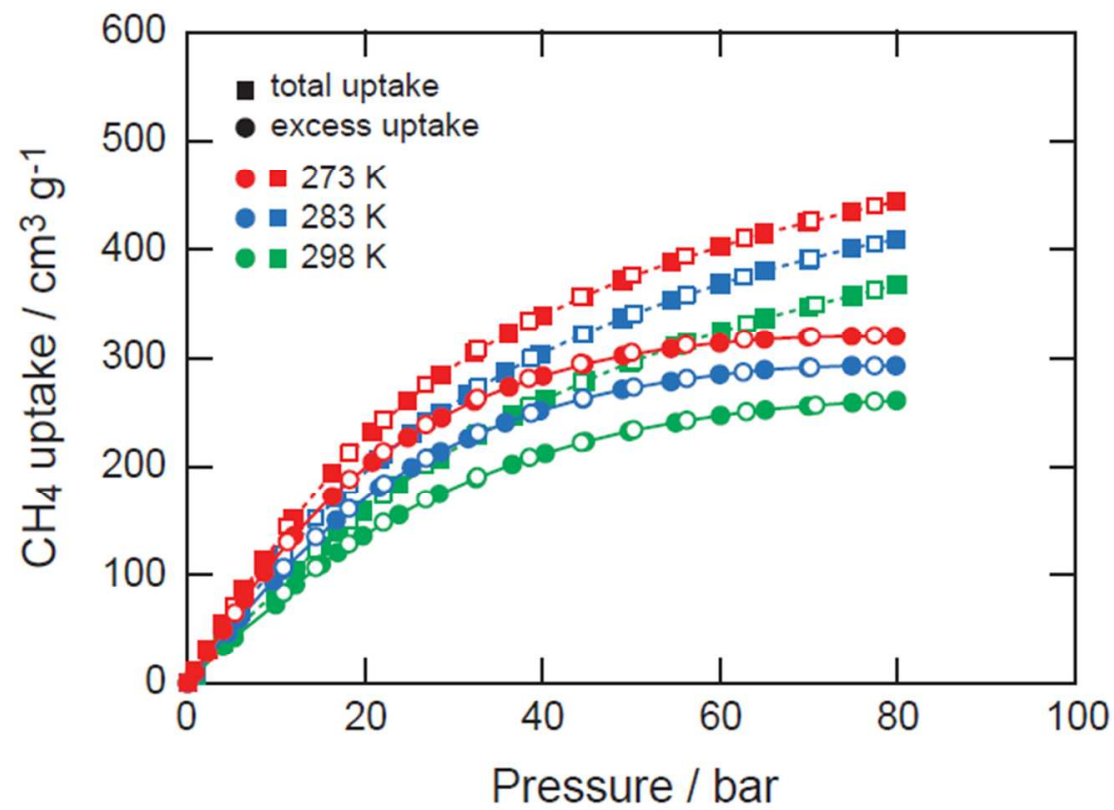

Figure S27 Excess and total $\mathrm{CH}_{4}$ adsorption isotherms for MOF-905- $\mathrm{NO}_{2}$ measured at 273, 283, and $298 \mathrm{~K}$.

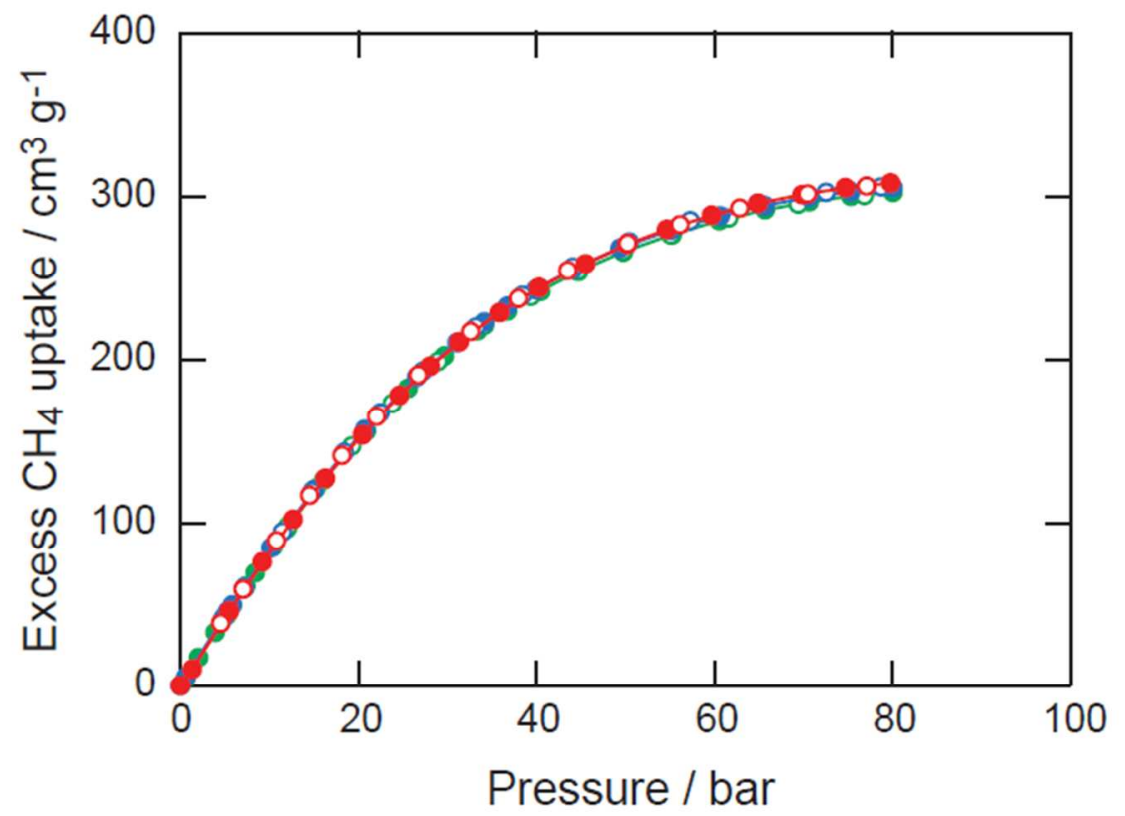

Figure S28. Excess $\mathrm{CH}_{4}$ adsorption isotherms for independently prepared MOF-905 measured at $298 \mathrm{~K}$. 
Section S8 Complete List of Metal-Organic Frameworks with ith-d Net

Table S6. Summary of crystallorgraphic and pore-structure properties of all MOFs with ith-d net.

\begin{tabular}{|c|c|c|c|c|c|c|}
\hline Material & $\begin{array}{l}\text { Space } \\
\text { Group }\end{array}$ & $\begin{array}{c}\text { Cell Parameter } \\
\AA\end{array}$ & $\begin{array}{l}\text { BET Surface area } \\
\qquad \mathrm{m}^{2} \mathrm{~g}^{-1}\end{array}$ & $\begin{array}{c}V_{\mathrm{p}} \\
\mathrm{cm}^{3} \mathrm{~g}^{-1}\end{array}$ & $\begin{array}{l}d_{\text {crystal }} \\
\mathrm{g} \mathrm{cm}^{-3}\end{array}$ & Ref. No. \\
\hline MOF-905 & $P m \overline{3}$ & 23.408 & 3490 & 1.34 & 0.549 & This Work \\
\hline MOF-905--Me 2 & $P m \overline{3}$ & 24.721 & 3640 & 1.39 & 0.568 & This Work \\
\hline MOF-905-Naph & $P m \overline{3}$ & 24.740 & 3310 & 1.25 & 0.585 & This Work \\
\hline MOF-905- $\mathrm{NO}_{2}$ & $P m \overline{3}$ & 23.992 & 3380 & 1.29 & 0.580 & This Work \\
\hline MOF-205 (DUT-6) & $P m \overline{3} n$ & 30.245 & 4530 & 2.16 & 0.380 & $S 1, S 2$ \\
\hline FJI-1 & $P m \overline{3} n$ & 27.736 & 4043 & 1.45 & 0.405 & $S 3$ \\
\hline \multirow{3}{*}{ DUT-23 (SUMOF-1) } & \multirow{3}{*}{$P m \overline{3} n$} & $27.725(\mathrm{Zn})$ & $4340(\mathrm{Zn})$ & $1.85(\mathrm{Zn})$ & $0.406(\mathrm{Zn})$ & \multirow{3}{*}{$S 4, S 5$} \\
\hline & & $27.634(\mathrm{Co})$ & $4850(\mathrm{Co})$ & $2.03(\mathrm{Co})$ & $0.403(\mathrm{Co})$ & \\
\hline & & $27.512(\mathrm{Cu})$ & $4730(\mathrm{Cu})$ & $2.01(\mathrm{Cu})$ & $0.413(\mathrm{Cu})$ & \\
\hline FJI-1-NH 2 & $P \overline{4} 3 n$ & 27.657 & N/A & N/A & 0.482 & S6 \\
\hline MUF-7a & $I \overline{4} 3 d$ & 60.232 & 4140 & 1.94 & 0.387 & $S 7$ \\
\hline MUF-7b & $I \overline{4} 3 d$ & 60.229 & 4480 & 2.15 & 0.392 & $S 7$ \\
\hline MUF-7c & $I \overline{4} 3 d$ & 60.233 & 3910 & 1.84 & 0.396 & $S 7$ \\
\hline
\end{tabular}




\begin{tabular}{lcccccc}
\hline MUF-7d & $I \overline{4} 3 d$ & 60.214 & 3790 & 1.84 & 0.401 & $S 7$ \\
MUF-7e & $I \overline{4} 3 d$ & 60.305 & 3910 & 1.84 & 0.429 & $S 7$ \\
MUF-7f & $I \overline{4} 3 d$ & 60.259 & 3000 & 1.30 & 0.435 & $S 7$ \\
MUF-7g & $I \overline{4} 3 d$ & 60.290 & 3820 & 1.74 & 0.438 & $S 7$ \\
MUF-7h & $I \overline{4} 3 d$ & 60.242 & 4000 & 1.93 & 0.444 & $S 7$ \\
MOF-205-(OH) 2 & $P m \overline{3} n$ & 30.367 & 4354 & 2.01 & 0.391 & $S 8$ \\
MOF-205-NH 2 & $P m \overline{3} n$ & 30.310 & 4330 & 0.83 & 0.387 & $S 9$ \\
MOF-205-NO 2 & $P m \overline{3} n$ & 30.312 & 3980 & 0.79 & 0.398 & $S 9$ \\
MOF-205-OBn & $P m \overline{3}$ & 30.173 & 3470 & 0.78 & 0.458 & $S 9$ \\
Zn ${ }_{4} \mathrm{O}(\mathrm{TPB})_{4 / 3}(\mathrm{BDC})$ & $P m \overline{3} n$ & 26.510 & 2874 & 1.20 & 0.561 & $S 10$ \\
MUF-77-methyl & $P m \overline{3}$ & 29.953 & 3600 & 1.85 & 0.452 & $S 11$ \\
MUF-77-ethyl & $P m \overline{3}$ & 29.951 & 3600 & 1.55 & 0.494 & $S 11$ \\
MUF-77-butyl & $P m \overline{3}$ & 29.936 & 3250 & 1.21 & 0.578 & $S 11$ \\
MUF-77-hexyl & $P m \overline{3}$ & 29.861 & 2170 & 1.00 & 0.667 & $S 11$ \\
MUF-77-octyl & $P m \overline{3}$ & 29.812 & 1570 & 0.65 & 0.754 & $S 11$ \\
MUF-77-decyl & $P m \overline{3}$ & 29.806 & 1170 & 0.48 & 0.839 & $S 11$ \\
\hline
\end{tabular}




\section{Section S9 References}

(S1) Klein, N.; Senkovska, I.; Gedrich, K.; Stoeck, U.; Henschel, A.; Mueller, U.; Kaskel, S. Angew. Chem. Int. Ed. 2009, 48, 9954-9957.

(S2) Furukawa, H.; Ko, N.; Go, Y. B.; Aratani, N.; Choi, S. B.; Choi, E.; Yazaydin, A. O.; Snurr, R. Q.; O'Keeffe, M.; Kim, J.; Yaghi, O. M. Science 2010, 329, 424-428.

(S3) Han, D.; Jiang, F.-L.; Wu, M.-Y.; Chen, L.; Chen, Q.-H.; Hong, M.-C. Chem. Commun. 2011, 47, 9861-9863.

(S4) Klein, N.; Senkovska, I.; Baburin, I. A.; Grünker, R.; Stoeck, U.; Schlichtenmayer, M.; Streppel, B.; Mueller, U.; Leoni, S.; Hirscher, M.; Kaskel, S. Chem. Eur. J. 2011, 17, 13007-13016.

(S5) Yao, Q.; Sun, J.; Li, K.; Su, J.; Peskov, M. V.; Zou, X. Dalton Trans. 2012, 41, 3953-3955.

(S6) Zhang, L.; Jian, Y.; Wang, J.; He, C.; Li, X.; Liu, T.; Duan, C. Dalton Trans. 2012, 41, 10153-10155.

(S7) Liu, L.; Konstas, K.; Hill, M. R.; Telfer, S. G. J. Am. Chem. Soc. 2013, 135, 17731-17734.

(S8) Spanopoulos, I.; Xydias, P.; Malliakas, C. D.; Trikalitis, P. N. Inorg. Chem. 2013, $52,855-862$.

(S9) Sim, J.; Yim, H.; Ko, N.; Choi, S. B.; Oh, Y.; Park, H. J.; Park, S. Y.; Kim, J. Dalton Trans. 2014, 43, 18017-18024.

(S10) Helten, S.; Sahoo, B.; Bon, V.; Senkovska, I.; Kaskel, S.; Glorius, F. CrystEngComm. 2015, 17, 307-312.

(S11) Liu, L.; Telfer, S. G. J. Am. Chem. Soc. 2015, 137, 3901-3909. 